\title{
A Look at Employment-Equity Groups Among Recent Postsecondary Graduates: Visible Minorities, Aboriginal Peoples and the Activity Limited by
} Ted Wannell, Nathalie Caron No. 69

\author{
11F0019MPE No.69 \\ ISBN: 0-662-22500-7 24th Floor R.H. Coats Building, Ottawa K1A 0T6
Voice: (613) 951-3546 FAX: 951-5643
Inquiries about this publication should be directed to Analytical Studies Branch (613) 951-1804 \\ Business and Labour Market Analysis Group, Statistics Canada
}

November 1994

The analysis presented in this paper is the responsibility of the authors and does not necessarily represent the view or policies of Statistics Canada

For: Labour Market Outlook and Sectoral Analysis Branch

Human Resources Development Canada 


\section{Explanation of Symbols}

The data presented in this paper originate from a sample survey and are therefore estimates of the target population figures. In general, the variability of the estimates increases as the sample size decreases. The relative variability of an estimate is measured by the coefficient of variation. Statistics Canada publication guidelines prohibit the publication of estimates with a coefficient of variation greater than 33 percent. In tables, these estimates are replaced with the symbol "..". Estimates with a coefficient in the range of 16.5 to 33 percent are published with a cautionary asterisk $(*)$, denoting their relatively high variability. 


\begin{abstract}
Employment equity legislation is becoming more prevalent in Canadian labour markets, yet -- other than broad availability numbers -- the labour market experiences of designated groups have not been well documented. Using the National Graduates Survey of 1992, this report profiles the early labour market experiences of visible minorities, Aboriginal peoples and persons with disabilities who graduated from Canadian universities and community colleges in 1990. In general, we find that the earnings of designated group members are very similar to the earnings of their classmates. However, we also find that members of these groups are more likely to be unemployed and are less likely to participate in the labour force than others in their class.
\end{abstract}

Key Words: earnings, wages, earnings gap, gender, employment equity, disabled persons, visible minorities, Aboriginal peoples, discrimination graduates, university, community college 


\section{Table of Contents}

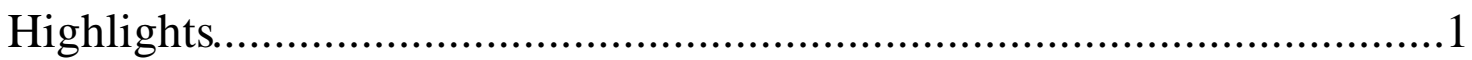

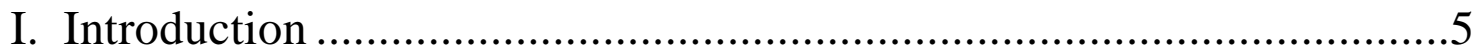

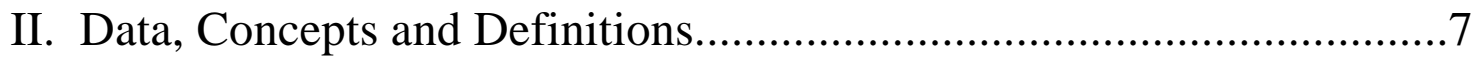

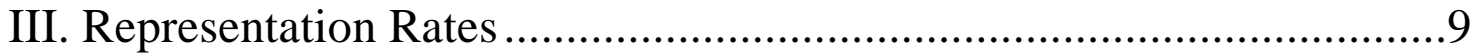

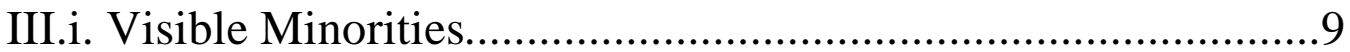

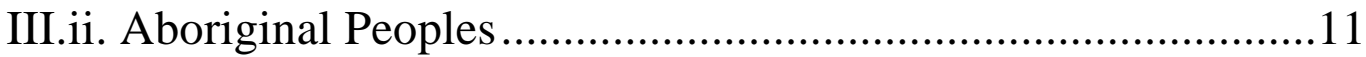

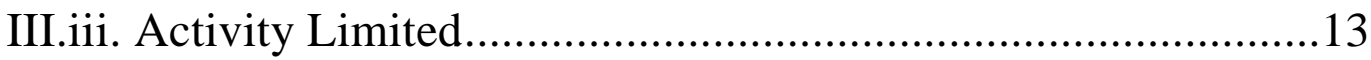

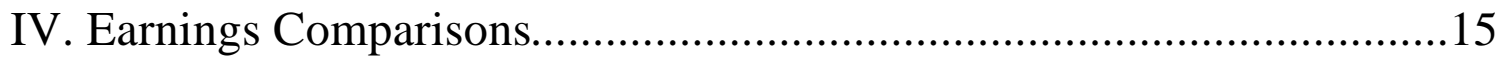

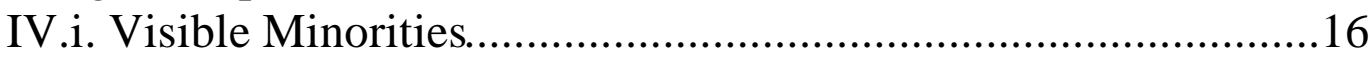

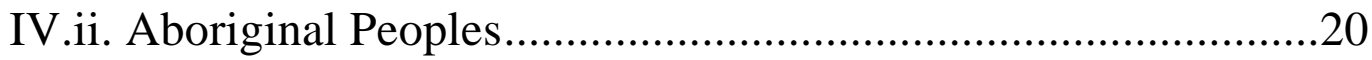

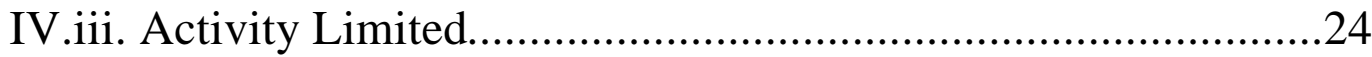

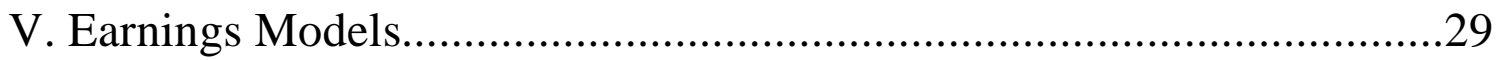

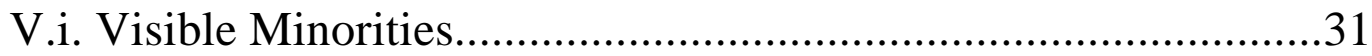

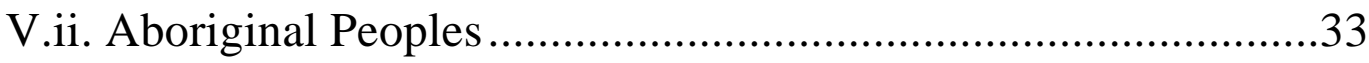

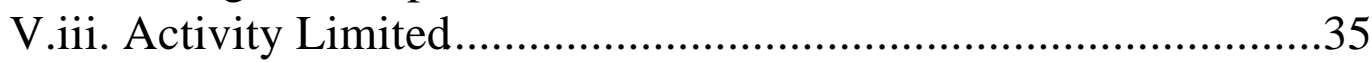

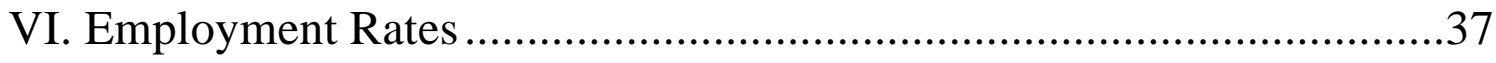

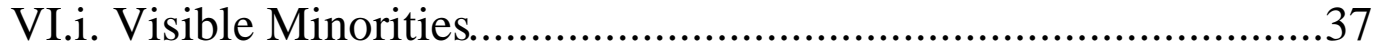

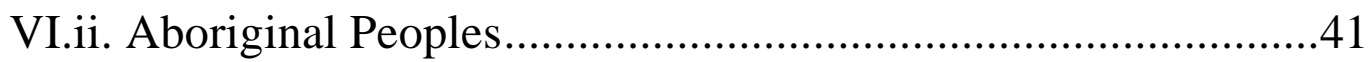

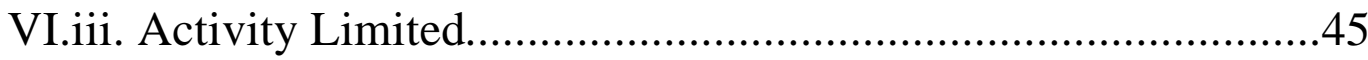

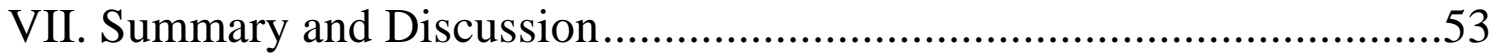

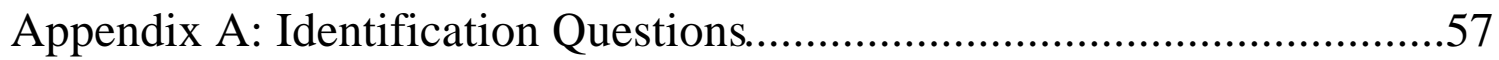

Appendix B: Industry and Occupation Coding........................................61 


\section{List of Tables}

Table 1. Visible Minorities Representation Among 1990 University

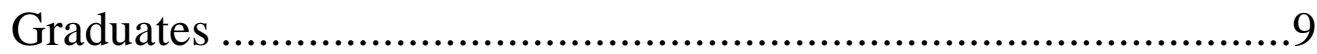

Table 2. Visible Minorities Representation Among 1990 Community College Graduates

Table 3. Aboriginal Peoples Representation Among 1990 University Graduates

Table 4. Aboriginal Peoples Representation Among 1990 Community College Graduates

Table 5. Activity-Limited Representation Among 1990 University Graduates

Table 6. Activity-Limited Representation Among 1990 Community College Graduates

Table 7. Average 1992 Earnings by Visible Minority Status, 1990 University Graduates (Full-time Workers, Earnings \$5,000 $\$ 500,000)$

Table 8. Average 1992 Earnings by Visible Minority Status, 1990

Community College Graduates (Full-time Workers, Earnings $\$ 5,000$ - $\$ 500,000)$

Table 9. Average 1992 Earnings by Aboriginal Peoples Status, 1990 University Graduates (Full-time Workers, Earnings \$5,000 $\$ 500,000)$

Table 10. Average 1992 Earnings by Aboriginal Peoples Status, 1990 Community College Graduates (Full-time Workers, Earnings $\$ 5,000$ - $\$ 500,000)$

Table 11. Average 1992 Earnings by Activity-Limited Status, 1990 University Graduates (Full-time Workers, Earnings \$5,000 $\$ 500,000)$

Table 12. Average 1992 Earnings by Activity-Limited Status, 1990 Community College Graduates (Full-time Workers, Earnings $\$ 5,000$ - $\$ 500,000)$

Table 13. Visible Minorities Earnings Model, 1990 University Graduates (Full-time at All Timepoints, Earnings \$5,000 $\$ 500,000)$

Table 14. Visible Minorities Earnings Model, 1990 Community College Graduates (Full-time at All Timepoints, Earnings $\$ 5,000$ - $\$ 500,000)$ 
Table 15. Aboriginal Peoples Earnings Model, 1990 University

Graduates (Full-time at All Timepoints, Earnings \$5,000 $\$ 500,000)$

Table 16. Aboriginal Peoples Earnings Model, 1990 Community

College Graduates (Full-time at All Timepoints, Earnings $\$ 5,000$ - $\$ 500,000)$

Table 17. Activity-Limited Earnings Model, 1990 University Graduates

(Full-time at All Timepoints, Earnings \$5,000 - \$500,000).

Table 18. Activity-Limited Earnings Model, 1990 Community College

Graduates (Full-time at All Timepoints, Earnings \$5,000 $\$ 500,000)$

Table 19. Labour Force Status (June 1992) by Visible Minority Status, 1990 University Graduates.

Table 20. Labour Force Participation Rate and Unemployment Rate (June 1992) by Visible Minority Status, 1990 University Graduates 38

Table 21. Labour Force Status (June 1992) by Visible Minority Status, 1990 Community College Graduates.

Table 22. Labour Force Participation Rate and Unemployment Rate (June 1992) by Visible Minority Status, 1990 Community College Graduates .40

Table 23. Labour Force Status (June 1992) by Aboriginal Peoples Status, 1990 University Graduates

Table 24. Labour Force Participation Rate and Unemployment Rate (June 1992) by Aboriginal Peoples Status, 1990 University Graduates

Table 25. Labour Force Status (June 1992) by Aboriginal Peoples

Status, 1990 Community College Graduates.

Table 26. Labour Force Participation Rate and Unemployment Rate (June 1992) by Aboriginal Peoples Status, 1990 Community College Graduates .44

Table 27. Labour Force Status (June 1992) by Activity-Limited Status, 1990 University Graduates.

Table 28. Labour Force Participation Rate and Unemployment Rate (June 1992) by Activity-Limited Status, 1990 University Graduates .46

Table 29. Labour Force Status (June 1992) by Activity-Limited Status, 1990 Community College Graduates. 
Table 30. Labour Force Participation Rate and Unemployment Rate (June 1992) by Activity-Limited Status, 1990 Community

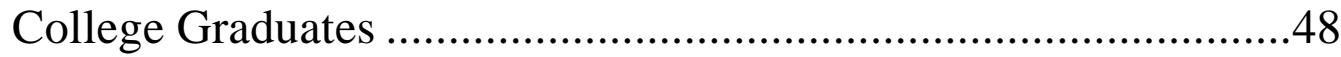





\section{HIGHLIGHTS}

\section{Visible Minorities}

The representation rate of visible minorities among 1990 postsecondary graduates is roughly proportional to their share of the broader population. More than ten percent of the 1990 graduates from universities and community colleges are members of visible minorities (the visible minority share of the 1991 population is nine-and-a-half percent). Visible minorities are particularly well-represented in science-based fields of study.

The earnings of visible minority graduates differ only slightly from the earnings of other graduates: among full-time workers, visible minority university graduates earned an average of two percent more than other graduates while visible minority community college graduates earned two percent less.

A multivariate earnings model was used in order to estimate whether visible minorities were rewarded the same as other graduates, controlling for population characteristics. The results are statistically significant, but account for less than one percent of average earnings. So holding population characteristics equal, visible minorities are rewarded about the same as their classmates: fractionally better among university graduates, fractionally worse among community college graduates.

In contrast, the employment rates for visible minorities are substantially lower than those of other graduates: by almost eight percentage points for university graduates and about six percentage points for community college graduates. These differences, which are due to both lower participation rates and higher unemployment rates for visible minority graduates, appear for graduates of most fields of study and in most regions (except British Columbia).

A multivariate model of employment for university graduates indicates that employment disparities are more acute for visible minority men than for visible minority women. Relative to other male graduates, unemployment was higher for visible minority members whose mother tongue was other than English or French, who were married or divorced and particularly those who graduated from a science-based field of study. Given the equality of earnings, it is difficult to cite the unemployment disparity as unambiguous evidence of hiring discrimination. Alternatively, employer adherence to tacit hiring quotas could produce similar results. 


\section{Aboriginal Peoples}

Aboriginal peoples are under-represented among postsecondary graduates relative to their presence in the overall population. While their share of the 1991 population is 3.8 percent, the representation rate of the Aboriginal peoples is only 1.2 percent among 1990 university graduates and 2.8 percent among community college graduates. At universities, Aboriginal peoples' representation is particularly low among advanced degree holders $(0.5$ percent).

Aboriginal peoples earn about the same as other graduates. A multivariate model estimated only negligible differences - about a tenth of a percent of average earnings -between the reward structure for Aboriginal peoples and other graduates.

While the employment situation for Aboriginal peoples does not differ greatly from that of other university graduates, a great disparity exists among community college graduates. Indeed, the unemployment rate for Aboriginal peoples is almost ten percentage points higher than for other community college graduates. In the absence of greater sample sizes and more geographical detail, it is impossible to determine why the unemployment rate is so high for Aboriginal community college graduates.

\section{Activity Limited}

The National Graduates Survey(NGS) uses a much shorter set of questions on activity limitation than the sequence used to produce official estimates of persons with disabilities. Consequently, we use the term activity limited rather than persons with disabilities in this report. According to the NGS sequence, just under four percent of university graduates and six-and-a-half percent of community college graduates are in some way limited in their activities at work, school or home. Persons with disabilities were estimated to comprise seven percent of the 1991 population.

The activity limited earned less than other graduates in 1992. The earnings gap was about seven percent among university graduates and one-and-a half percent among community college graduates. However, the multivariate earnings models do not provide strong evidence of differential treatment for activity-limited graduates.

Among university graduates, the employment rate of the activity limited is six percentage points lower than for other graduates. For community college graduates, this gap in the employment rate rises to twelve percent. On average, activity-limited graduates experience both lower participation rates and higher unemployment rates than other graduates. In contrast to other areas of the country, university and community college graduates with activity limitations had lower unemployment rates in Alberta than others in their class. This was also the case for university graduates in Manitoba. 
To explore the notion that employment problems might be related to the severity of a handicap, we constructed a crude index of the severity of an individual's limitations. Although labour force participation tended to fall as the index increased, the unemployment rate was fairly consistent across all index levels. This seems to indicate that even those with relatively minor limitations have more difficulty in finding a job than other graduates. 
- 4 - 


\section{Introduction}

The Employment Equity Act of 1986 established the goal of workplace equality for women, visible minorities, Aboriginal peoples and persons with disabilities. While the Act is primarily concerned with the representation of designated groups in the workplace, the general notion of equality encompasses much more. It is the tacit assumption of this paper that equality in the labour market can be measured as the labour market rewards -in terms of earnings and employment -- to investments in education and training.

This report focuses on a small segment of the population: 1990 graduates of Canadian universities and community colleges. Attention is further limited to visible minorities, Aboriginal peoples and persons with disabilities. Gender issues are covered in a separate paper. ${ }^{1}$ The issues involved in identifying members of the designated groups are covered in the first section of the report. The second section presents the representation rates for each of the designated groups in the class of 1990. The third section provides a descriptive comparison of the earnings of designated groups and the earnings of other graduates. A more rigorous, multivariate comparison of earnings follows. Next is an examination of the employment, participation and unemployment rates of designated groups compared to others. Finally, the results for each designated group are summarized and discussed.

The main finding of these analyses is quite clear. Although there is little difference between the earnings of the designated groups and the remainder of the population, members of each designated group (except Aboriginal university graduates) were less likely to be employed than other postsecondary graduates. Accordingly, designated group members were over-represented among both the unemployed and those not-in-the-labourforce.

\footnotetext{
${ }^{1}$ Wannell and Caron. The Gender Earnings Gap Among Recent Postsecondary Graduates, 1984-92.
} Statistics Canada. 1994. 
- 6 - 


\section{Data, Concepts and Definitions}

This report is based on data from the 1992 National Graduates Survey(NGS). The survey population is representative of the 1990 graduates of Canadian universities, community colleges and trade/vocational schools. Due to the small sample size of trade/vocational graduates, the analyses are limited to university and community college graduates. The data file contains information on 19,284 university respondents and 14,794 community college respondents, representing total graduate populations of 126,266 and 90,908 , respectively.

The question of whom to include in each of the designated groups is addressed in the Employment Equity Regulations that accompany the Employment Equity Act. ${ }^{2}$ The regulations outline the following concepts:

Visible Minority - $\quad$ persons, other than Aboriginal peoples, who are non-Caucasian in race and non-white in colour.

Aboriginal peoples - persons who are Indians, Inuit or Métis.

Persons with Disabilities - persons who:

(i) have any persistent physical, mental, psychiatric, sensory or learning impairment;

(ii) consider themselves to be, or believe that an employer or a potential employer would be likely to consider them to be, disadvantaged in employment by reason of an impairment referred to in subparagraph (i).

The concepts outlined in the regulations are operationalized by an Interdepartmental Working Group on Employment Equity Data. Data on visible minorities and Aboriginal peoples come from the Census of the Population, while data on persons with disabilities come from the Health and Activity Limitation Survey (HALS).

Visible minorities are identified primarily by the Census ethnicity question. This question asks: "To which ethnic or cultural group(s) did (the respondent's) ancestors belong?". The Working Group has prepared a list of responses that corresponds as closely as possible to the conceptual definition (see Appendix A). Note that respondents may provide more than one ethnicity response -- this is termed multiple response. In the case of multiple response, a person is classified as a member of a visible minority if any of their responses matches one on the list. The ethnicity information is supplemented by information on places of birth (e.g. Haiti) or first languages (e.g. Hindi) that likely indicate visible minority status.

\footnotetext{
${ }^{2}$ Women, Visible Minorities, Aboriginal Peoples and Persons with Disabilities: The 1991 Employment Equity Definitions. The Interdepartmental Working Group on Employment Equity Data. December, 1993.
} 
Aboriginal peoples are similarly identified by the Census ethnicity question. Thus any single or multiple response referring to North American Indian, Inuit, Métis or a specific First Nation or band name leads to an Aboriginal peoples designation.

As noted above, persons with disabilities are identified through HALS. HALS is a post-censal survey of census respondents designed to collect data on the nature and severity of disabilities, as well as the problems associated with disabilities. HALS disability status is determined through a sequence of approximately 15 questions (see Appendix A). The HALS sample is partially stratified according to a screening sequence of four questions on the Census of the Population . Approximately 20 percent of those identified as potentially disabled by the Census screening sequence fail to conform to the HALS disability definition. ${ }^{3}$ Conversely, about five percent of those who answer no to all the Census screening questions are subsequently identified as disabled by HALS.

The questions used to identify designated group status on the 1992 National Graduates Survey do not conform precisely to the operational definitions as outlined by the Interdepartmental Working Group.

One difference relates to the wording of the NGS ethnicity question. While the Census question asks about the cultural or ethnic background of the respondent's ancestors, the NGS question asks about the background of the respondent. Overall, this apparently minor difference has a large impact on the level of multiple ethnicity response -18 percent on the NGS compared to 32 percent for a similar group of Census respondents ${ }^{4}$. Paradoxically, the multiple response rate is higher for visible minorities in the NGS (16 percent) than in the Census (13 percent).

The other difference between the Census and NGS coding of visible minority status is in the use of country of birth and mother tongue to supplement the ethnicity question. As noted above, the Census uses these variables to pick up some visible minority members who may be missed by the ethnicity question. This option is not available on the NGS since mother tongue is not captured at a detailed level for languages other than English or French and place of birth is not captured at all. Since these steps account for some five percent of the visible minorities identified in the Census, NGS estimates of visible minority representation are likely lower by a similar factor.

With respect to persons with disabilities, the NGS uses the four Census screening questions rather than the much longer HALS screening sequence. While this makes sense in the context that the NGS was already a very long survey, it leads to estimates of persons with disabilities that would differ substantially from the official HALS definition. In recognition of this difference, we use the term activity limited in this paper rather than persons with disabilities.

\footnotetext{
${ }^{3}$ Health and Activity Limitation Survey - 1991 User's Guide. Post-Censal Surveys Program, Statistics Canada. 1993.

${ }^{4}$ The Census comparison group consists of university and community college graduates reweighted to have approximately the same age distribution as the NGS respondents.
} 


\section{Representation Rates}

Designated groups' representation rates are calculated by dividing the number in the designated group in a population by the total population. Non-response and responses for which the designated status is unknown (e.g. ethnicity = "other") are not included in the calculations.

\section{III.i. Visible Minorities}

Table 1. Visible Minorities Representation Among 1990 University Graduates

\begin{tabular}{|c|c|c|c|}
\hline & $\begin{array}{c}\text { Visible } \\
\text { Minority }\end{array}$ & $\begin{array}{l}\text { Not Visible } \\
\text { Minonity }\end{array}$ & $\begin{array}{c}\text { Visible } \\
\text { Minority Rate }\end{array}$ \\
\hline Total & 11905 & 101047 & $10.5 \%$ \\
\hline \multicolumn{4}{|l|}{ Degree Level } \\
\hline Bachelors / 1st Prof. & 9706 & 87010 & $10.0 \%$ \\
\hline Masters & 1844 & 12470 & $12.9 \%$ \\
\hline Doctorate & 353 & 1480 & $19.3 \%$ \\
\hline \multicolumn{4}{|l|}{ Field of Study } \\
\hline None/Unknown & $473 *$ & 3941 & $10.7 \% *$ \\
\hline Education & 799 & 18572 & $4.1 \%$ \\
\hline Fine Arts \& Humanities & 1332 & 15643 & $7.8 \%$ \\
\hline Commerce, Economics \& Law & 2376 & 20179 & $10.5 \%$ \\
\hline Other Social Sciences & 1589 & 18659 & $7.8 \%$ \\
\hline Agricultural \& Biological Sciences & 1197 & 5959 & $16.7 \%$ \\
\hline Engineering & 1678 & 6262 & $21.1 \%$ \\
\hline Medical \& Other Health & 1104 & 6567 & $14.4 \%$ \\
\hline Math \& Physical Sciences & 1357 & 5265 & $20.5 \%$ \\
\hline \multicolumn{4}{|l|}{ Province / Region } \\
\hline Atlantic Provinces & 466 & 11356 & $3.9 \%$ \\
\hline Quebec & 2115 & 26276 & $7.5 \%$ \\
\hline Ontario & 5844 & 41281 & $12.4 \%$ \\
\hline Manitoba & 427 & 3747 & $10.2 \%$ \\
\hline Saskatchewan & 228 & 4105 & $5.3 \%$ \\
\hline Alberta & 1107 & 7167 & $13.4 \%$ \\
\hline British Columbia & 1719 & 7116 & $19.5 \%$ \\
\hline
\end{tabular}

Ten-and-a-half percent of 1990 university graduates are members of visible minorities. The visible minority representation rate increases with degree level - from 10 percent at the undergraduate level to 19 percent at the $\mathrm{PhD}$ level. Visible minorities were far more prevalent in natural sciences and engineering than in the arts and social sciences. 
The representation rate, in general, rises from east to west, ranging from 4 percent in the Atlantic Provinces to almost 20 percent in British Columbia.

Table 2. Visible Minorities Representation Among 1990 Community College Graduates

\begin{tabular}{|c|c|c|c|}
\hline & $\begin{array}{c}\text { Visible } \\
\text { Minority }\end{array}$ & $\begin{array}{l}\text { Not Visible } \\
\text { Minority }\end{array}$ & $\begin{array}{c}\text { Visible } \\
\text { Minority Rate }\end{array}$ \\
\hline Total & 8403 & 72764 & $10.4 \%$ \\
\hline \multicolumn{4}{|l|}{ Diploma Type } \\
\hline Tra de Certific ate / Diploma & 3894 & 26286 & $12.9 \%$ \\
\hline College Certificate / Diploma & 4509 & 46458 & $8.8 \%$ \\
\hline \multicolumn{4}{|l|}{ Field of Study } \\
\hline Arts \& Huma nities & 1623 & 10006 & $14.0 \%$ \\
\hline Health Sciences & 1535 & 15166 & $9.2 \%$ \\
\hline Other Engineering Technology & 633 & 4112 & $13.3 \%$ \\
\hline $\begin{array}{l}\text { Electronics, Math \& Computer } \\
\text { Science }\end{array}$ & 567 & 4011 & $12.4 \%$ \\
\hline $\begin{array}{l}\text { Mechanic al \& Structural Engineering } \\
\text { Tech. }\end{array}$ & 374 & 5007 & $7.0 \%$ \\
\hline Natura I Sc ienc es \& Prima ry Ind ustries & $317 *$ & 4794 & $6.2 \% *$ \\
\hline Social Sciences and Services & 944 & 9748 & $8.8 \%$ \\
\hline $\begin{array}{l}\text { Sec reta rial Sciences \& } \\
\text { Merchandising }\end{array}$ & 448 & 5313 & $7.8 \%$ \\
\hline Management \& Adminstration & 1303 & 11170 & $10.4 \%$ \\
\hline Miscoded & 660 & 3436 & $16.1 \%$ \\
\hline \multicolumn{4}{|l|}{ Province } \\
\hline Atlantic Provinces & 68 & 5279 & $1.3 \%$ \\
\hline Quebec & 715 & 15222 & $4.5 \%$ \\
\hline Ontario & 4134 & 28385 & $12.7 \%$ \\
\hline Manitoba & 208 & 2226 & $8.5 \%$ \\
\hline Saskatc hewan & $44 *$ & 2292 & $1.9 \% *$ \\
\hline Alberta & 810 & 7083 & $10.3 \%$ \\
\hline British Columbia \& Temitories & 2424 & 12277 & $16.5 \%$ \\
\hline
\end{tabular}

Members of visible minorities comprise 10.4 percent of the 1990 graduating class of community colleges. The visible minority representation rate is much higher for trade and vocational programs (13 percent) than for programs that lead to a community college certificate or diploma (about 9 percent). In contrast to the university graduates, there is no clear split between the representation rates in arts as opposed to science and technology. Among community college graduates, visible minority representation is lowest in the Atlantic provinces and Saskatchewan and highest in British Columbia. 


\section{III.ii. Aboriginal Peoples}

Table 3. Aboriginal Peoples Representation Among 1990 University Graduates

\begin{tabular}{||l|c|c|c||}
\hline \hline & $\begin{array}{c}\text { Aboriginal } \\
\text { Peoples }\end{array}$ & $\begin{array}{c}\text { Not Aboriginal } \\
\text { Peoples }\end{array}$ & $\begin{array}{c}\text { Representation } \\
\text { Rate }\end{array}$ \\
\hline Total & $\mathbf{1 , 5 6 8}$ & $\mathbf{1 2 4 , 4 6 7}$ & $\mathbf{1 . 2 \%}$ \\
Degree Level & & & \\
Bachelors/ 1st Professional & 1,469 & 106,379 & $\mathbf{1 . 4 \%}$ \\
Masters/Doctorate & 99 & 18,079 & $\mathbf{0 . 5 \%}$ \\
Field of Study & & & \\
Education & $370 *$ & 21,129 & $\mathbf{1 . 7 \%} *$ \\
Fine Arts, Humanities & $230 *$ & 18,637 & $\mathbf{1 . 2 \%} *$ \\
Commerce, Economics, Law & $147 *$ & 24,587 & $\mathbf{0 . 6 \%} *$ \\
Other Social Sciences & $441 *$ & 22,666 & $\mathbf{1 . 9 \%} * \|$ \\
Sciences & $290 *$ & 32,635 & $\mathbf{0 . 9 \%} *$ \\
Province / Region & & & \\
Atlantic Provinces & $141 *$ & 11,936 & $\mathbf{1 . 2 \%} *$ \\
Quebec & $268 *$ & 29,706 & $\mathbf{0 . 9 \%} *$ \\
Ontario & $504 *$ & 53,362 & $\mathbf{0 . 9 \%} * \|$ \\
Manitoba & $154 *$ & 5,170 & $\mathbf{2 . 9 \%} *$ \\
Saskatchewan & 247 & 4,803 & $\mathbf{4 . 9 \%}$ \\
Alberta & $142 *$ & 9,572 & $\mathbf{1 . 5 \%} * \|$ \\
British Columbia & $113 *$ & 9,919 & $\mathbf{1 . 1 \%} *$ \\
\hline \hline
\end{tabular}

Just over one percent of 1990 university graduates indicated Aboriginal peoples status, quite low compared to Aboriginal peoples' 3.8 percent share of the total population and 3 percent share of the workforce population. Aboriginal peoples' representation is particularly low -- half of a percent -- among advanced degree graduates. By field of study, the percentage of Aboriginal peoples ranges from 0.6 percent in Commerce, Economics and Law to 1.7 percent in Education. Universities in Saskatchewan (4.9 percent) and Manitoba (2.9 percent) have the highest percentages of Aboriginal peoples graduates. 
Table 4. Aboriginal Peoples Representation Among 1990 Community College Graduates

\begin{tabular}{|c|c|c|c|}
\hline & $\begin{array}{l}\text { Aborigin } \\
\text { al } \\
\text { Peoples }\end{array}$ & $\begin{array}{l}\text { Not } \\
\text { Aboriginal } \\
\text { Peoples }\end{array}$ & $\begin{array}{l}\text { Representation } \\
\text { Rate }\end{array}$ \\
\hline Total & 2560 & 88348 & $2.8 \%$ \\
\hline \multicolumn{4}{|l|}{ Diploma Type } \\
\hline Tra de Certific ate / Diploma & 1334 & 33006 & $3.9 \%$ \\
\hline College Certificate / Diploma & 1226 & 55322 & $2.2 \%$ \\
\hline \multicolumn{4}{|l|}{ Field of Study } \\
\hline Arts \& Huma nities & $321 *$ & 12664 & $2.5 \% *$ \\
\hline Health Sciences & 507 & 18219 & $2.7 \%$ \\
\hline $\begin{array}{l}\text { Electronics, Comp Sci, Math, Other Eng. } \\
\text { Tech }\end{array}$ & $176 *$ & 10278 & $1.7 \% *$ \\
\hline $\begin{array}{l}\text { Mechanic al \& Structural Engineering } \\
\text { Tech. }\end{array}$ & $208 *$ & 5987 & $3.4 \% *$ \\
\hline Na tura I Sc iences \& Primary Ind ustries & $208 *$ & 5495 & $3.6 \% *$ \\
\hline Social Sciences and Services & 431 & 11446 & $3.6 \%$ \\
\hline Sec reta ria I Sc iences \& Merc ha nd ising & $122 *$ & 6279 & $1.9 \% *$ \\
\hline Management \& Adminstration & 452 & 13551 & $3.2 \%$ \\
\hline \multicolumn{4}{|l|}{ Province } \\
\hline Atlantic Provinces & $93 *$ & 5343 & $1.7 \% *$ \\
\hline Quebec & $173 *$ & 16380 & $1.0 \%$ * \\
\hline Ontario & 897 & 35495 & $2.5 \%$ \\
\hline Manitoba & 199 & 2805 & $6.6 \%$ \\
\hline Saskatchewan & 198 & 2599 & $7.1 \%$ \\
\hline Alberta & 342 & 9299 & $3.5 \%$ \\
\hline British Columbia, Yukon & 503 & 16351 & $3.0 \%$ \\
\hline Northwest Ternitories & 155 & 77 & $66.8 \%$ \\
\hline
\end{tabular}

The Aboriginal peoples representation rate among 1990 community college graduates ( 3 percent) is more than double the rate among university graduates. At the community colleges, Aboriginal peoples tend to be concentrated in trade and vocational programs rather than in diploma or certificate programs. By field of study, the representation rate ranges from a low of 1.7 percent in Electronics, Computer Science, Math and Other Engineering Technologies to highs of 3.6 percent in Natural Sciences and Primary Industries and Social Sciences and Services. Fully two-thirds of community college graduates in the Northwest Territories indicate Aboriginal peoples origins. Elsewhere, Aboriginal peoples comprise from one percent (Quebec) to 7 percent (Saskatchewan) of community college graduates. 


\section{III.iii. Activity Limited}

Table 5. Activity-Limited Representation Among 1990 University Graduates

\begin{tabular}{|c|c|c|c|}
\hline & $\begin{array}{l}\text { Activity } \\
\text { Limited } \\
\end{array}$ & $\begin{array}{c}\text { Not Activity } \\
\text { Limited } \\
\end{array}$ & $\begin{array}{l}\text { Representation } \\
\text { Rate } \\
\end{array}$ \\
\hline Total & 4,875 & 121,160 & $3.9 \%$ \\
\hline \multicolumn{4}{|l|}{ Degree Level } \\
\hline Bachelors / 1st Prof. & 4,238 & 103,611 & $3.9 \%$ \\
\hline Masters & 574 & 15,521 & $3.6 \%$ \\
\hline Doctorate & 63 & 2,020 & $3.0 \%$ \\
\hline \multicolumn{4}{|l|}{ Field of Study } \\
\hline None / Unknown & $222 *$ & 4,681 & $4.5 \%$ * \\
\hline Education & 754 & 20,745 & $3.5 \%$ \\
\hline Fine Arts \& Humanities & 972 & 17,895 & $5.2 \%$ \\
\hline Commerce, Economics \& Law & 817 & 23,918 & $3.3 \%$ \\
\hline Other Social Sciences & 1,102 & 22,004 & $4.8 \%$ \\
\hline Agricultural \& Biological Sciences & $183 *$ & 7,816 & $2.3 \%$ * \\
\hline Engineering & $200 *$ & 8,726 & $2.2 \%$ * \\
\hline Medical \& Other Health & $376 *$ & 8,189 & $4.4 \%$ * \\
\hline Math \& Physical Sciences & $249 *$ & 7,187 & $3.3 \%$ * \\
\hline \multicolumn{4}{|l|}{ Province } \\
\hline Newfoundland & 126 & 2,274 & $5.2 \%$ \\
\hline Prince Edward Island & $24 *$ & 420 & $5.5 \%$ * \\
\hline Nova Scotia & 243 & 5,799 & $4.0 \%$ \\
\hline New Brunswick & 125 & 3,064 & $3.9 \%$ \\
\hline Quebec & $620 *$ & 29,355 & $2.1 \%$ * \\
\hline Ontario & 2,071 & 51,795 & $3.8 \%$ \\
\hline Manitoba & 287 & 5,038 & $5.4 \%$ \\
\hline Saskatchewan & 274 & 4,775 & $5.4 \%$ \\
\hline Alberta & 613 & 9,101 & $6.3 \%$ \\
\hline British Columbia & 493 & 9,538 & $4.9 \%$ \\
\hline
\end{tabular}

Nearly 4 percent of 1990 university graduates are in some way limited in their activities at work, school or home. This compares to an estimate of seven percent among the 1991 population as a whole and six-and-a-half percent among the workforce. 5 The representation of activity-limited individuals drops slightly with each step up in degree level. Among fields of study, Engineering has the lowest rate of activity-limited graduates (2.2 percent), while Fine Arts and Humanities has the highest (5.2 percent).

${ }^{5}$ These estimates are based on the 1991 Census screening sequence which served as the model for the NGS screening sequence. The Health and Activity Limitations Survey is the source of official estimates of persons with disabilities. 
Geographically, the activity-limited representation rate ranges from 2.1 percent in Quebec to 6.3 percent in Alberta.

Table 6. Activity-Limited Representation Among 1990 Community College Graduates

\begin{tabular}{|c|c|c|c|}
\hline & $\begin{array}{l}\text { Activity } \\
\text { Limited }\end{array}$ & $\begin{array}{l}\text { Not Activity } \\
\text { Limited }\end{array}$ & $\begin{array}{l}\text { Representation } \\
\text { Rate }\end{array}$ \\
\hline Total & 5,887 & 85,021 & $6.5 \%$ \\
\hline \multicolumn{4}{|l|}{ Diploma Type } \\
\hline Trade Certificate / Diploma & 3,029 & 31,310 & $8.8 \%$ \\
\hline College Certificate / Diploma & 2,858 & 53,691 & $5.1 \%$ \\
\hline \multicolumn{4}{|l|}{ Field of Study } \\
\hline Arts \& Humanities & 1,007 & 11,979 & $7.8 \%$ \\
\hline Health Sciences & 1,056 & 17,670 & $5.6 \%$ \\
\hline Other Engineering Technology & $318 *$ & 5,091 & $5.9 \% *$ \\
\hline Electronics, Math \& Computer Science & $246 *$ & 4,799 & $4.9 \% *$ \\
\hline Mechanical \& Structural Engineering Tech. & 442 & 5,753 & $7.1 \%$ \\
\hline Natural Sciences \& Primary Industries & 403 & 5,300 & $7.1 \%$ \\
\hline Social Sciences and Services & 848 & 11,029 & $7.1 \%$ \\
\hline Secretarial Sciences \& Merchandising & $305 *$ & 6,095 & $4.8 \% *$ \\
\hline Management \& Administration & 873 & 13,130 & $6.2 \%$ \\
\hline Miscoded & 388 & 4,175 & $8.5 \%$ \\
\hline \multicolumn{4}{|l|}{ Province / Region } \\
\hline Newfoundland & 88 & 1,257 & $6.6 \%$ \\
\hline Prince Edward Island & $36 *$ & 758 & $4.6 \% *$ \\
\hline Nova Scotia & $44 *$ & 660 & $6.3 \% *$ \\
\hline New Brunswick & 159 & 2,434 & $6.1 \%$ \\
\hline Quebec & $423 *$ & 16,130 & $2.6 \% *$ \\
\hline Ontario & 2,293 & 34,099 & $6.3 \%$ \\
\hline Manitoba & 256 & 2,748 & $8.5 \%$ \\
\hline Saskatchewan & 185 & 2,611 & $6.6 \%$ \\
\hline Alberta & 804 & 8,836 & $8.3 \%$ \\
\hline British Columbia, Territories & 1,599 & 15,488 & $9.4 \%$ \\
\hline
\end{tabular}

Persons with activity limitations are more prevalent among community college than university graduates, accounting for six-and-a-half percent of the 1990 graduating class. Within the community colleges, activity-limited persons are more likely to graduate from trade and vocational programs than diploma or certificate programs. Those with activity limitations are fairly evenly spread across fields of study, with representation rates ranging from 4.8\% in Secretarial Sciences and Merchandising to 7.8 percent in Arts and Humanities. The rate is also fairly consistent across provinces, with the exception of Quebec which has a much lower than average percentage of activity-limited graduates. 


\section{Earnings Comparisons}

In the National Graduates Survey, respondents are asked to estimate their yearly earnings based on working at the job they held in the week prior to the survey for an entire year. To control for the possibility that differential rates of part-time employment might affect the earnings comparisons, we limit the population to full-time workers (i.e. those who worked more than 30 hours in the reference week). An inspection of the earnings of full-time workers revealed a number of responses at the extreme high and low ends of the earnings scale. By cross-checking these responses against industry, occupation and previous year income, we judged that nearly all values outside the range of $\$ 5,000$ to $\$ 500,000$ were coding or response error. As such, we excluded these out-of-range values from the tabulations.

The earnings ratio expresses the average earnings of the designated group as a percentage of the average earnings of other graduates. If both groups earn the same, the ratio would be 100 . If the designated group earns less, the ratio will be less than 100; if more, greater than 100 .

For the earnings comparisons, there are no restrictions on multiple designated group status. For example, when the earnings of Aboriginal peoples are compared to all others, both groups will include women, activity-limited persons and visible minorities (to the extent that there is multiple response overlap between Aboriginal peoples and visible minorities).

Some variation in the category by category earnings ratio is to be expected. While consistent large differences may be indicative of differential treatment, it is important to recognize that earnings are the result of a multitude of factors. As such, it is inappropriate to interpret a low earnings ratio within a single category of industry, occupation or field of study as evidence of discrimination. Conversely, a high earnings ratio should not be construed as "reverse discrimination". To simultaneously control for the many factors that influence earnings, a multivariate model is presented in section $\mathrm{V}$. 


\section{IV.i. Visible Minorities}

Table 7. Average 1992 Earnings by Visible Minority Status, 1990 University Graduates (Full-time Workers, Earnings \$5,000 - \$500,000)

\begin{tabular}{|c|c|c|c|c|c|}
\hline \multirow[b]{2}{*}{ Characteristics } & \multicolumn{2}{|c|}{$\begin{array}{l}\text { Weighted } \\
\text { Number }\end{array}$} & \multicolumn{2}{|c|}{$\begin{array}{l}\text { Average } \\
\text { Eamings }\end{array}$} & \multirow{2}{*}{\begin{tabular}{|l} 
Eamings \\
Ratio
\end{tabular}} \\
\hline & $\begin{array}{c}\text { Visible } \\
\text { Minonity }\end{array}$ & $\begin{array}{r}\text { Not Visible } \\
\text { Minority }\end{array}$ & $\begin{array}{c}\text { Visible } \\
\text { Minonity }\end{array}$ & $\begin{array}{r}\text { Not Visible } \\
\text { Minority }\end{array}$ & \\
\hline & & & $\$ \prime 000$ & $\$ \prime 000$ & $\%$ \\
\hline Total & 7118 & 68233 & 35.8 & 35.1 & 101.9 \\
\hline \multicolumn{6}{|l|}{ Highest Degree J une 1992} \\
\hline Bachelors / 1st Prof. & 5565 & 54949 & 33.9 & 33.1 & 102.5 \\
\hline Masters & 1243 & 11961 & 42.6 & 43.2 & 98.7 \\
\hline Doctorate & 310 & 1277 & 42.9 & 49.7 & 86.5 \\
\hline \multicolumn{6}{|l|}{ Occupation } \\
\hline Managers & 603 & 7757 & 40.3 & 39.7 & 101.6 \\
\hline Managerial Related & 1007 & 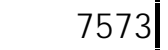 & 36.2 & 35.9 & 101.0 \\
\hline Physical \& Life Sciences & $198 *$ & 1572 & $32.7 *$ & 33.4 & $97.7 *$ \\
\hline Arc hitec ture \& Eng ineering & 684 & 3912 & 38.7 & 37.5 & 103.4 \\
\hline Math \& Computer Science & 727 & 2917 & 38.1 & 39.0 & 97.7 \\
\hline Social Sc iences \& Religion & $457 *$ & 7894 & $33.2 *$ & 36.3 & $91.5 *$ \\
\hline University Tea ching & $315 *$ & 1768 & $29.0 *$ & 32.5 & $89.2 *$ \\
\hline Other Teaching & 605 & 14548 & 37.9 & 36.3 & 104.5 \\
\hline Health Diagnosis & $299 *$ & 1673 & $59.6 *$ & 47.4 & $125.7 *$ \\
\hline Nursing, Other Health & $424 *$ & 4253 & $43.3 *$ & 36.9 & $117.3 *$ \\
\hline Arts \& Recreation & $154 *$ & 1994 & $31.0 *$ & 26.9 & $115.3 *$ \\
\hline Clerical & 870 & 4384 & 24.6 & 25.0 & 98.5 \\
\hline Sales & $278 *$ & 3429 & $34.0 *$ & 31.4 & $108.3 *$ \\
\hline Service Occupations & $218 *$ & 2077 & $24.7 *$ & 27.5 & $89.9 *$ \\
\hline Blue Collar & $266 *$ & 2456 & $32.1 *$ & 28.1 & $114.1 *$ \\
\hline
\end{tabular}

On average, visible minorities earn slightly more than other university graduates. However, the earnings of visible minorities don't rise as much at higher degree levels, so they average less than other graduates at the masters and doctoral level. In most occupations, visible minorities earn about the same as other graduates. There are a couple of occupations where they earn appreciably less than others (University Teaching and Service Occupations) and several where they earn appreciably more (Health Occupations and Arts \& Recreation). 
Table 7. Average 1992 Earnings by Visible Minority Status, 1990 University Graduates (Full-time Workers, Earnings \$5,000 - \$500,000) - completed

\begin{tabular}{|c|c|c|c|c|c|}
\hline \multirow[b]{2}{*}{ Characteristics } & \multicolumn{2}{|c|}{$\begin{array}{l}\text { Weighted } \\
\text { Number }\end{array}$} & \multicolumn{2}{|c|}{$\begin{array}{l}\text { Average } \\
\text { Earnings }\end{array}$} & \multirow[t]{2}{*}{\begin{tabular}{|c|} 
Earnings \\
Ratio
\end{tabular}} \\
\hline & $\begin{array}{l}\text { Visible } \\
\text { Minority }\end{array}$ & $\begin{array}{c}\text { Not Visible } \\
\text { Minority }\end{array}$ & $\begin{array}{l}\text { Visible } \\
\text { Minority }\end{array}$ & $\begin{array}{c}\text { Not Visible } \\
\text { Minority }\end{array}$ & \\
\hline Industry & & & $\$ ’ 000$ & $\$>000$ & \\
\hline Primary Industries & .. & 1,388 & .. & 35.5 & .. \\
\hline Manufacturing \& Construction & 787 & 6,676 & 35.3 & 34.6 & 102.1 \\
\hline Transport, Communications \& Utilities & $483 *$ & 2,756 & $37.9 *$ & 37.3 & $101.8 *$ \\
\hline Wholesale Trade & $207 *$ & 1,521 & $32.2 *$ & 31.8 & $101.2 *$ \\
\hline Retail \& Consumer Services & $536 *$ & 5,857 & $30.0 *$ & 27.3 & $110.1 *$ \\
\hline Finance & 626 & 2,510 & 30.2 & 35.0 & 86.3 \\
\hline Insurance \& Real Estate & $256 *$ & 1,897 & $32.8 *$ & 35.7 & $91.9 *$ \\
\hline Education & 1,151 & 18,625 & 35.0 & 36.5 & 95.8 \\
\hline Health & 905 & 9,069 & 45.3 & 36.9 & 122.9 \\
\hline Welfare \& Religion & $185 *$ & 1,344 & $29.7 *$ & 28.3 & $104.9 *$ \\
\hline Services to Business Management & 1,200 & 9,456 & 36.8 & 35.9 & 102.4 \\
\hline Public Administration & 685 & 7,122 & 34.7 & 36.3 & 95.5 \\
\hline Field of Study & & & & & \\
\hline None / Unknown & $238 *$ & 2,395 & $28.1 *$ & 33.3 & $84.5 *$ \\
\hline Education & $571 *$ & 13,215 & $40.2 *$ & 37.2 & $108.1 *$ \\
\hline Fine Arts \& Humanities & $583 *$ & 8,468 & $30.3 *$ & 28.7 & $105.5 *$ \\
\hline Commerce, Economics \& Law & 1,581 & 15,340 & 36.5 & 37.8 & 96.7 \\
\hline Other Social Sciences & 977 & 11,477 & 30.3 & 31.9 & 95.0 \\
\hline Agricultural \& Biological Sciences & $539 *$ & 3,662 & $28.0 *$ & 29.3 & $95.5 *$ \\
\hline Engineering & 1,076 & 4,781 & 36.9 & 38.3 & 96.4 \\
\hline Medical \& Other Health & 744 & 5,142 & 49.5 & 42.2 & 117.2 \\
\hline Math \& Physical Sciences & 810 & 3,754 & 35.5 & 35.0 & 101.5 \\
\hline
\end{tabular}

Visible minority graduates working in Finance, Insurance and Real Estate industries earn somewhat less, on average, than other graduates, while those in health industries earn somewhat more. In line with health industries and occupations, the Medical \& Other Health field of study also shows elevated earnings for visible minority graduates. Visible minority graduates earn significantly less than others where there is no field of study specialization. 
Table 8. Average 1992 Earnings by Visible Minority Status, 1990 Community College Graduates (Full-time Workers, Earnings \$5,000 - \$500,000)

\begin{tabular}{|c|c|c|c|c|c|}
\hline \multirow[b]{2}{*}{ Characteristics } & \multicolumn{2}{|c|}{$\begin{array}{l}\text { Weighted } \\
\text { Number }\end{array}$} & \multicolumn{2}{|c|}{$\begin{array}{l}\text { Average } \\
\text { Earnings }\end{array}$} & \multirow[t]{2}{*}{$\begin{array}{c}\text { Earnings } \\
\text { Ratio } \\
\end{array}$} \\
\hline & $\begin{array}{l}\text { Visible } \\
\text { Minority }\end{array}$ & $\begin{array}{c}\text { Not Visible } \\
\text { Minority }\end{array}$ & $\begin{array}{l}\text { Visible } \\
\text { Minority }\end{array}$ & $\begin{array}{c}\text { Not Visible } \\
\text { Minority }\end{array}$ & \\
\hline & & & $\$ ’ 000$ & $\$ \prime 000$ & \\
\hline Total & 4,730 & 46,757 & 26.1 & 26.5 & 98.2 \\
\hline Certificate Type & & & & & \\
\hline Trade Certificate or Diploma & 1,391 & 12,288 & 22.6 & 24.7 & 91.4 \\
\hline College Certificate or Diploma & 2,663 & 31,742 & 26.7 & 27.0 & 99.0 \\
\hline Occupation & & & & & \\
\hline Managers & $342 *$ & 3,250 & $28.8 *$ & 27.7 & $103.7^{*}$ \\
\hline Managerial Related & $306 *$ & 2,560 & $26.8 *$ & 26.3 & 102.1 * \\
\hline Physical \& Life Sciences &.. & 790 &.. & 27.1 &.. \\
\hline Architecture \& Engineering & $211 *$ & 2,027 & $28.7 *$ & 28.7 & 99.8 * \\
\hline Math \& Computer Science & $223 *$ & 1,555 & $33.8 *$ & 31.0 & $109.1 *$ \\
\hline Social Sciences \& Religion & $149 *$ & 1,888 & $26.5 *$ & 26.4 & $100.0 *$ \\
\hline University Teaching &.. & 95 &.. & 27.7 &.. \\
\hline Other Teaching & $94 *$ & 1,586 & $23.3 *$ & 24.2 & $96.0 *$ \\
\hline Health Diagnosis &.. & 159 &.. & 28.8 & .. \\
\hline Nursing, Other Health & 734 & 7,766 & 30.4 & 30.7 & 99.2 \\
\hline Arts \& Recreation & .. & 1,297 & .. & 24.2 & .. \\
\hline Clerical & 920 & 7,526 & 22.9 & 22.2 & 103.1 \\
\hline Sales & $138 *$ & 2,374 & $26.2 *$ & 23.5 & 111.6 \\
\hline Service Occupations & 447 & 4,124 & 19.9 & 24.3 & 82.1 \\
\hline Blue Collar & 1,069 & 9,714 & 25.6 & 27.4 & 93.6 \\
\hline
\end{tabular}

Members of a visible minority earn, on average, slightly less than other community college graduates. The gap is somewhat larger for trade and vocational programs than diploma and certificate programs. The gap varies only modestly by occupation, with the exception of Service Occupations -- where visible minorities earn an average of 18 percent less than other graduates. 
Table 8. Average 1992 Earnings by Visible Minority Status, 1990 Community College Graduates (Full-time Workers, Earnings \$5,000 - \$500,000)

- completed

\begin{tabular}{|c|c|c|c|c|c|}
\hline \multirow[b]{2}{*}{ Characteristics } & \multicolumn{2}{|c|}{$\begin{array}{l}\text { Weighted } \\
\text { Number }\end{array}$} & \multicolumn{2}{|c|}{$\begin{array}{l}\text { Average } \\
\text { Earnings }\end{array}$} & \multirow[t]{2}{*}{$\begin{array}{c}\text { Earnings } \\
\text { Ratio } \\
\end{array}$} \\
\hline & $\begin{array}{l}\text { Visible } \\
\text { Minority }\end{array}$ & $\begin{array}{c}\text { Not Visible } \\
\text { Minority }\end{array}$ & $\begin{array}{l}\text { Visible } \\
\text { Minority }\end{array}$ & $\begin{array}{c}\text { Not Visible } \\
\text { Minority }\end{array}$ & \\
\hline & & & $\$ ’ 000$ & $\$>000$ & \\
\hline Industry & & & & & \\
\hline Primary Industries & .. & 1,645 & .. & 28.2 & .. \\
\hline Manufacturing \& Construction & 962 & 7,942 & 25.8 & 27.3 & 94.5 \\
\hline Transport, Communications \& Utilities & $323 *$ & 3,180 & $30.4 *$ & 29.3 & $103.7^{*}$ \\
\hline Wholesale Trade & $172 *$ & 1,801 & $23.1 *$ & 24.8 & 93.4 * \\
\hline Retail \& Consumer Services & 756 & 7,484 & 20.7 & 21.2 & 97.8 \\
\hline Finance & $166 *$ & 1,212 & $24.9 *$ & 24.2 & $102.9^{*}$ \\
\hline Insurance \& Real Estate & $201 *$ & 1,094 & $27.7 *$ & 25.2 & $109.9^{*}$ \\
\hline Education & $143 *$ & 1,771 & $26.9 *$ & 26.9 & 100.0 * \\
\hline Health & 1,110 & 11,275 & 28.1 & 28.7 & 97.7 \\
\hline Welfare \& Religion & .. & 389 &.. & 24.7 & .. \\
\hline Services to Business Management & 486 & 4,392 & 26.1 & 24.7 & 105.4 \\
\hline Public Administration & $285 *$ & 4,549 & $30.3 *$ & 29.3 & 103.4 * \\
\hline Field of Study & & & & & \\
\hline Arts \& Humanities & 851 & 5,830 & 25.1 & 25.0 & 100.4 \\
\hline Health Sciences & 871 & 9,087 & 28.7 & 29.7 & 96.8 \\
\hline Other Engineering Technology & $363 *$ & 2,916 & $26.5 *$ & 28.4 & 93.3 * \\
\hline Electronics, Math \& Computer Science & $365 *$ & 3,044 & $26.1 *$ & 27.2 & 95.9 * \\
\hline Mechanical \& Structural Engineering Tech. & $217 *$ & 3,421 & $26.9 *$ & 29.1 & 92.4 * \\
\hline Natural Sciences \& Primary Industries & $224 *$ & 2,950 & $27.9 *$ & 25.8 & $108.3^{*}$ \\
\hline Social Sciences and Services & 494 & 6,534 & 25.7 & 26.4 & 97.1 \\
\hline Secretarial Sciences \& Merchandising & $271 *$ & 3,566 & $24.5 *$ & 24.1 & 101.5 * \\
\hline Management \& Administration & 772 & 7,341 & 24.5 & 23.1 & 106.0 \\
\hline Miscoded & $301 *$ & 2,067 & $24.6 *$ & 26.9 & 91.2 * \\
\hline
\end{tabular}

The earnings of visible minorities and other community college graduates do not differ greatly by industry or field of study. All ratios in these categories are within ten percent of equality. 


\section{IV.ii. Aboriginal Peoples}

Table 9. Average 1992 Earnings by Aboriginal Peoples Status, 1990 University Graduates (Full-time Workers, Earnings \$5,000 - \$500,000)

\begin{tabular}{|c|c|c|c|c|c|}
\hline \multirow[b]{2}{*}{ Characteristics } & \multicolumn{2}{|c|}{$\begin{array}{l}\text { Weighted } \\
\text { Number }\end{array}$} & \multicolumn{2}{|c|}{$\begin{array}{l}\text { Average } \\
\text { Earnings }\end{array}$} & \multirow[t]{2}{*}{$\begin{array}{l}\text { Earnings } \\
\text { Ratio }\end{array}$} \\
\hline & $\begin{array}{c}\text { Aboriginal } \\
\text { Peoples }\end{array}$ & $\begin{array}{c}\text { Not } \\
\text { Aboriginal } \\
\text { Peoples }\end{array}$ & $\begin{array}{l}\text { Aboriginal } \\
\text { Peoples }\end{array}$ & $\begin{array}{c}\text { Not } \\
\text { Aboriginal } \\
\text { Peoples }\end{array}$ & \\
\hline & & & $\$ ’ 000$ & $\${ }^{\prime} 000$ & \\
\hline Total & 1,052 & 82,564 & 36.1 & 35.2 & 102.5 \\
\hline Highest Degree June 1992 & & & & & \\
\hline Bachelors / 1st Prof. & 895 & 66,428 & 34.7 & 33.1 & 104.7 \\
\hline Masters / Doctorate & 157 & 16,136 & 44.3 & 43.3 & 102.4 \\
\hline Occupation & & & & & \\
\hline Managers & $150 *$ & 9,081 & $41.5 *$ & 39.8 & 104.1 * \\
\hline Managerial Related &.. & 9,236 &.. & 35.3 & .. \\
\hline Physical \& Life Sciences & .. & 1,967 & .. & 33.7 & .. \\
\hline Architecture \& Engineering & .. & 5,074 & .. & 37.5 & .. \\
\hline Math \& Computer Science & .. & 4,098 & .. & 38.5 & .. \\
\hline Social Sciences \& Religion & $158 *$ & 9,073 & $34.9 *$ & 36.4 & 95.8 * \\
\hline University Teaching &.. & 2,294 &.. & 32.2 & .. \\
\hline Other Teaching & $246 *$ & 16,475 & $35.6 *$ & 36.5 & 97.4 * \\
\hline Health Diagnosis &.. & 2,185 &.. & 51.0 & .. \\
\hline Nursing, Other Health & .. & 5,084 & .. & 37.4 & .. \\
\hline Arts \& Recreation & .. & 2,305 & .. & 27.0 & .. \\
\hline Clerical & .. & 5,876 & .. & 24.9 & .. \\
\hline Sales & .. & 4,134 & .. & 31.1 &.. \\
\hline Service Occupations & .. & 2,599 & .. & 27.3 & .. \\
\hline Blue Collar & .. & 3,043 &.. & 28.7 &.. \\
\hline
\end{tabular}

The relatively small number of Aboriginal peoples among 1990 university graduates makes it difficult to produce reliable estimates of earnings for most subgroups. Overall, Aboriginal peoples earn slightly higher average salaries than other graduates. 
Table 9. Average 1992 Earnings by Aboriginal Peoples Status, 1990 University Graduates (Full-time Workers, Earnings \$5,000 - \$500,000) - completed

\begin{tabular}{|c|c|c|c|c|c|}
\hline & \multicolumn{2}{|c|}{$\begin{array}{l}\text { Weighted } \\
\text { Number }\end{array}$} & \multicolumn{2}{|c|}{$\begin{array}{l}\text { Average } \\
\text { Earnings }\end{array}$} & \multirow[t]{2}{*}{$\begin{array}{l}\text { Earnings } \\
\text { Ratio }\end{array}$} \\
\hline Characteristics & $\begin{array}{c}\text { Aboriginal } \\
\text { Peoples }\end{array}$ & $\begin{array}{c}\text { Not } \\
\text { Aboriginal } \\
\text { Peoples }\end{array}$ & $\begin{array}{l}\text { Aboriginal } \\
\text { Peoples }\end{array}$ & $\begin{array}{c}\text { Not } \\
\text { Aboriginal } \\
\text { Peoples }\end{array}$ & \\
\hline Industry & & & $\$ ’ 000$ & $\$ ’ 000$ & \\
\hline Primary Industries & .. & 1,632 & .. & 36.1 & .. \\
\hline Manufacturing \& Construction & .. & 8,272 & .. & 34.7 & .. \\
\hline Transport, Communications \& Utilities & .. & 3,604 &.. & 37.2 & .. \\
\hline Wholesale Trade & .. & 1,947 & .. & 32.3 & .. \\
\hline Retail \& Consumer Services & .. & 7,601 & .. & 26.8 & .. \\
\hline Finance & .. & 3,379 & .. & 34.1 &.. \\
\hline Insurance \& Real Estate & $299 *$ & 2,466 & $34.8 *$ & 34.9 & 99.5 * \\
\hline Education & $187 *$ & 21,580 & $32.5 *$ & 36.5 & 89.0 * \\
\hline Health &.. & 10,930 &.. & 38.0 &.. \\
\hline Welfare \& Religion &.. & 974 & .. & 30.2 & .. \\
\hline Services to Business Management & $176 *$ & 11,583 & $37.6 *$ & 35.8 & 105.1 * \\
\hline Public Administration &.. & 8,582 &.. & 36.2 &.. \\
\hline Field of Study & & & & & \\
\hline Education & $304 *$ & 15,008 & $40.6 *$ & 37.2 & 109.1 * \\
\hline Fine Arts \& Humanities & $154 *$ & 9,724 & $31.1 *$ & 28.6 & 108.6 * \\
\hline Commerce, Economics \& Law & .. & 18,420 & .. & 37.7 & .. \\
\hline Other Social Sciences & $252 *$ & 13,849 & $31.3 *$ & 31.7 & 98.8 * \\
\hline Agricultural \& Biological Sciences &.. & 4,620 &.. & 29.1 & .. \\
\hline Engineering & .. & 6,576 & .. & 37.9 & .. \\
\hline Medical \& Other Health & .. & 6,446 & .. & 43.8 & .. \\
\hline Math \& Physical Sciences & .. & 5,058 & .. & 35.0 & .. \\
\hline
\end{tabular}


Table 10. Average 1992 Earnings by Aboriginal Peoples Status, 1990 Community College Graduates (Full-time Workers, Earnings \$5,000 - \$500,000)

\begin{tabular}{|c|c|c|c|c|c|}
\hline & \multicolumn{2}{|c|}{$\begin{array}{l}\text { Weighted } \\
\text { Number }\end{array}$} & \multicolumn{2}{|c|}{$\begin{array}{l}\text { Average } \\
\text { Eamings }\end{array}$} & \multirow[t]{2}{*}{$\begin{array}{l}\text { Earnings } \\
\text { Ratio }\end{array}$} \\
\hline Characteristics & $\begin{array}{l}\text { Aboriginal } \\
\text { Peoples }\end{array}$ & $\begin{array}{c}\text { Not Aboriginal } \\
\text { Peoples }\end{array}$ & $\begin{array}{l}\text { Aboriginal } \\
\text { Peoples }\end{array}$ & $\begin{array}{l}\text { Not Aboriginal } \\
\text { Peoples }\end{array}$ & \\
\hline & & & $\$ ’ 000$ & $\$, 000$ & $\%$ \\
\hline Total & 1352 & 56396 & 26.6 & 26.6 & 100.0 \\
\hline Certificate Type & & & & & \\
\hline Trade Certificate or Diploma & 512 & 15169 & 25.7 & 24.7 & 104.0 \\
\hline College Certificate or Diploma & 789 & 37498 & 27.0 & 27.0 & 100.1 \\
\hline Occupation & & & & & \\
\hline Managers & .. & 3895 & .. & 27.7 & .. \\
\hline Managerial Related & .. & 3128 & .. & 26.3 & .. \\
\hline Physical \& Life Sciences & $105 *$ & 876 & $28.4 *$ & 27.7 & 102.5 * \\
\hline Architecture \& Engineering &.. & 2479 &.. & 28.8 &.. \\
\hline Math \& Computer Science & .. & 1909 & .. & 31.1 & .. \\
\hline Social Sciences \& Religion & .. & 2165 & .. & 28.1 & .. \\
\hline University Teaching & .. & 105 & .. & 27.7 &.. \\
\hline Other Teaching & $133 *$ & 1846 & $28.5 *$ & 23.8 & 120.0 * \\
\hline Health Diagnosis &.. & 174 & .. & 31.8 &.. \\
\hline Nursing, Other Health & .. & 9388 & .. & 30.6 & .. \\
\hline Arts \& Recreation & $137 *$ & 1548 & $29.1 *$ & 24.0 & $121.3^{*}$ \\
\hline Clerical &.. & 9099 &.. & 22.3 & .. \\
\hline Sales & $263 *$ & 2793 & $21.2 *$ & 23.7 & 89.5 * \\
\hline Service Occupations &.. & 4950 &.. & 23.5 &.. \\
\hline Blue Collar & $146 *$ & 11995 & $29.8 *$ & 27.4 & $108.9^{*}$ \\
\hline
\end{tabular}

On average, Aboriginal peoples earn the same as other community college graduates. While the earnings ratio apparently swings widely among occupations, the sample sizes in each case are relatively small -- leading to unstable estimates. 
Table 10. Average 1992 Earnings by Aboriginal Peoples Status, 1990 Community College Graduates (Full-time Workers, Earnings \$5,000 - \$500,000) - completed

\begin{tabular}{|c|c|c|c|c|c|}
\hline \multirow[b]{2}{*}{ Characteristics } & \multicolumn{2}{|c|}{$\begin{array}{l}\text { Weighted } \\
\text { Number }\end{array}$} & \multicolumn{2}{|c|}{$\begin{array}{l}\text { Average } \\
\text { Earnings }\end{array}$} & \multirow[t]{2}{*}{$\begin{array}{c}\text { Earnings } \\
\text { Ratio }\end{array}$} \\
\hline & $\begin{array}{l}\text { Aboriginal } \\
\text { Peoples }\end{array}$ & $\begin{array}{c}\begin{array}{c}\text { Not Aboriginal } \\
\text { Peoples }\end{array} \\
\end{array}$ & $\begin{array}{l}\text { Aboriginal } \\
\text { Peoples }\end{array}$ & $\begin{array}{l}\text { Not Aboriginal } \\
\text { Peoples }\end{array}$ & \\
\hline & & & $\$ \mathbf{S}^{\prime} 000$ & $\$ \mathbf{S}^{\prime} 00$ & \\
\hline $\begin{array}{l}\text { Industry } \\
\text { Primary Industries }\end{array}$ & $310 *$ & 1,880 & $25.8 *$ & 27.9 & 92.4 * \\
\hline Manufacturing \& Construction &.. & 9,807 &.. & 27.4 &.. \\
\hline Transport, Communications \& Utilities & $203 *$ & 3,893 & $25.9 *$ & 29.6 & 87.6 * \\
\hline Wholesale Trade &.. & 2,269 & .. & 24.8 &.. \\
\hline Retail \& Consumer Services & .. & 9,265 & .. & 21.1 & .. \\
\hline Finance & $170 *$ & 1,509 & $17.0 *$ & 24.1 & 70.5 * \\
\hline Insurance \& Real Estate &.. & 1,424 &.. & 25.5 &.. \\
\hline Education & .. & 2,061 & .. & 25.8 & .. \\
\hline Health & .. & 13,566 & .. & 29.0 &.. \\
\hline Welfare \& Religion & $320 *$ & 393 & $27.2 *$ & 25.5 & 106.7 * \\
\hline Services to Business Management &.. & 5,268 &.. & 24.9 & .. \\
\hline Public Administration & .. & 5,038 & .. & 29.5 & .. \\
\hline Field of Study & & & & & \\
\hline Arts \& Humanities & $93 *$ & 7,362 & $23.6 *$ & 24.8 & 95.3 * \\
\hline Health Sciences & $256 *$ & 11,025 & $24.9 *$ & 29.9 & 83.1 * \\
\hline Other Engineering Technology & .. & 3,708 &.. & 28.3 &.. \\
\hline Electronics, Math \& Computer Science &.. & 3,715 & .. & 27.2 & .. \\
\hline $\begin{array}{l}\text { Mechanical \& Structural Engineering } \\
\text { Tech. }\end{array}$ & $137 *$ & 4,028 & $35.8 *$ & 28.9 & 123.9 * \\
\hline Natural Sciences \& Primary Industries & $107 *$ & 3,410 & $25.2 *$ & 26.4 & 95.5 * \\
\hline Social Sciences and Services & $256 *$ & 7,477 & $28.9 *$ & 26.2 & 110.3 * \\
\hline Secretarial Sciences \& Merchandising &.. & 4,217 &.. & 24.1 &.. \\
\hline Management \& Administration & $252 *$ & 8,897 & $23.6 *$ & 23.4 & 100.8 * \\
\hline Miscoded & .. & 2,558 &.. & 26.7 &.. \\
\hline
\end{tabular}

Again the earnings ratio bounces up and down among industries and fields of study, but should be interpreted cautiously due to the small sample sizes within categories. 


\section{IV.iii. Activity Limited}

Table 11. Average 1992 Earnings by Activity-Limited Status, 1990 University Graduates (Full-time Workers, Earnings \$5,000 - \$500,000)

\begin{tabular}{|c|c|c|c|c|c|}
\hline \multirow[t]{2}{*}{ Characteristics } & \multicolumn{2}{|c|}{$\begin{array}{l}\text { Weighted } \\
\text { Number }\end{array}$} & \multicolumn{2}{|c|}{$\begin{array}{l}\text { Average } \\
\text { Earnings }\end{array}$} & \multirow[t]{2}{*}{$\begin{array}{c}\text { Earnings } \\
\text { Ratio }\end{array}$} \\
\hline & $\begin{array}{l}\text { Activity } \\
\text { Limited }\end{array}$ & $\begin{array}{l}\text { Not Activity } \\
\text { Limited }\end{array}$ & $\begin{array}{l}\text { Activity } \\
\text { Limited }\end{array}$ & $\begin{array}{c}\text { Not Activity } \\
\text { Limited }\end{array}$ & \\
\hline & & & $\$ ’ 000$ & $\$ ’ 000$ & \\
\hline Total & 2,843 & 80,773 & 32.9 & 35.3 & 93.2 \\
\hline Highest Degree June 1992 & & & & & \\
\hline Bachelors / 1st Prof. & 2,276 & 65,047 & 30.6 & 33.2 & 92.1 \\
\hline Masters & 508 & 13,988 & 40.8 & 43.4 & 94.1 \\
\hline Doctorate & 59 & 1,737 & 54.2 & 48.0 & 112.9 \\
\hline Occupation & & & & & \\
\hline Managers & $254 *$ & 8,976 & $40.2 *$ & 39.8 & 101.1 * \\
\hline Managerial Related & $266 *$ & 9,087 & $32.5 *$ & 35.6 & 91.1 * \\
\hline Physical \& Life Sciences &.. & 1,936 & .. & 33.7 & .. \\
\hline Architecture \& Engineering & $194 *$ & 4,910 & $34.6 *$ & 37.6 & $92.0 *$ \\
\hline Math \& Computer Science & $171 *$ & 3,983 & $33.3 *$ & 38.8 & $85.8 *$ \\
\hline Social Sciences \& Religion & $446 *$ & 8,786 & $31.9 *$ & 36.6 & $87.1 *$ \\
\hline University Teaching &.. & 2,237 &.. & 32.3 &.. \\
\hline Other Teaching & $547 *$ & 16,175 & $36.5 *$ & 36.5 & 100.1 * \\
\hline Health Diagnosis &.. & 2,162 &.. & 50.9 &.. \\
\hline Nursing, Other Health & $255 *$ & 4,910 & $37.5 *$ & 37.3 & 100.4 * \\
\hline Arts \& Recreation &.. & 2,161 &.. & 27.4 &.. \\
\hline Clerical & $229 *$ & 5,689 & $23.0 *$ & 25.0 & 91.9 * \\
\hline Sales &.. & 4,103 &.. & 31.0 & .. \\
\hline Service Occupations & .. & 2,568 & .. & 27.2 & .. \\
\hline Blue Collar & .. & 3,051 & .. & 28.7 & .. \\
\hline
\end{tabular}

University graduates who indicated they have some activity limitation earned an average of seven percent less than other graduates. While the activity limited earned eight percent less at the undergraduate level and six percent less at the masters level, they earned 12 percent more at the doctoral level. The average earnings of the activity limited equaled that of other graduates in Management, Other Teaching (i.e. elementary and secondary) and Nursing \& Other Health, but was lower in all other occupational groups. 
Table 11. Average 1992 Earnings by Activity-Limited Status, 1990 University Graduates (Full-time Workers, Earnings \$5,000 - \$500,000) - completed

\begin{tabular}{|c|c|c|c|c|c|}
\hline \multirow[t]{2}{*}{ Characteristics } & \multicolumn{2}{|c|}{$\begin{array}{l}\text { Weighted } \\
\text { Number }\end{array}$} & \multicolumn{2}{|c|}{$\begin{array}{l}\text { Average } \\
\text { Earnings }\end{array}$} & \multirow[t]{2}{*}{$\begin{array}{l}\text { Earnings } \\
\text { Ratio }\end{array}$} \\
\hline & $\begin{array}{l}\text { Activity } \\
\text { Limited }\end{array}$ & $\begin{array}{l}\text { Not Activity } \\
\text { Limited }\end{array}$ & $\begin{array}{l}\text { Activity } \\
\text { Limited }\end{array}$ & $\begin{array}{l}\text { Not Activity } \\
\text { Limited }\end{array}$ & \\
\hline & & & $\$ ’ 000$ & $\$>00$ & \\
\hline Industry & & & & & \\
\hline Primary Industries & .. & 1,604 & .. & 36.0 & .. \\
\hline Manufacturing \& Construction & $192 *$ & 8,194 & $32.9 *$ & 34.9 & 94.2 * \\
\hline Transport, Communications \& Utilities &.. & 3,614 &.. & 37.2 &.. \\
\hline Wholesale Trade & .. & 1,905 & .. & 32.2 & .. \\
\hline Retail \& Consumer Services & $322 *$ & 7,387 & $23.4 *$ & 27.1 & $86.4 *$ \\
\hline Finance & .. & 3,273 & .. & 34.1 &.. \\
\hline Insurance \& Real Estate & .. & 2,462 & .. & 34.9 & .. \\
\hline Education & 766 & 21,113 & 35.0 & 36.5 & 95.9 \\
\hline Health & $547 *$ & 10,571 & $34.3 *$ & 38.1 & $90.0 *$ \\
\hline Welfare \& Religion &.. & 937 &.. & 30.3 &.. \\
\hline Services to Business Management & $469 *$ & 11,178 & $31.1 *$ & 36.0 & $86.3 *$ \\
\hline Public Administration & $238 *$ & 8,520 & $38.6 *$ & 36.2 & 106.8 * \\
\hline Field of Study & & & & & \\
\hline None / Unknown &.. & 2,826 & .. & 32.6 & .. \\
\hline Education & $495 *$ & 14,816 & $37.5 *$ & 37.3 & $100.5 *$ \\
\hline Fine Arts \& Humanities & $409 *$ & 9,470 & $23.6 *$ & 28.9 & $81.7 *$ \\
\hline Commerce, Economics \& Law & 637 & 17,880 & 33.7 & 37.8 & 89.2 \\
\hline Other Social Sciences & $537 *$ & 13,564 & $29.6 *$ & 31.8 & $93.2 *$ \\
\hline Agricultural \& Biological Sciences &.. & 4,587 &.. & 29.0 &.. \\
\hline Engineering & $150 *$ & 6,467 & $35.9 *$ & 38.0 & $94.5 *$ \\
\hline Medical \& Other Health & $295 *$ & 6,237 & $39.8 *$ & 43.9 & $90.7 *$ \\
\hline Math \& Physical Sciences & $170 *$ & 4,926 & $33.8 *$ & 35.1 & $96.3 *$ \\
\hline
\end{tabular}

Activity-limited graduates earned lower average salaries in every industry except Public Administration and every field of study except education. 
Table 12. Average 1992 Earnings by Activity-Limited Status, 1990 Community College Graduates (Full-time Workers, Earnings \$5,000 - \$500,000)

\begin{tabular}{|c|c|c|c|c|c|}
\hline & \multicolumn{2}{|c|}{$\begin{array}{l}\text { Weighted } \\
\text { Number }\end{array}$} & \multicolumn{2}{|c|}{$\begin{array}{l}\text { Average } \\
\text { Earnings }\end{array}$} & $\begin{array}{l}\text { Earnings } \\
\text { Ratio }\end{array}$ \\
\hline & $\begin{array}{l}\text { Activity } \\
\text { Limited }\end{array}$ & $\begin{array}{l}\text { Not Activity } \\
\text { Limited }\end{array}$ & $\begin{array}{l}\text { Activity } \\
\text { Limited }\end{array}$ & $\begin{array}{l}\text { Not Activity } \\
\text { Limited }\end{array}$ & \\
\hline & & & $\$ ’ 000$ & $\$>000$ & \\
\hline Total & 2,940 & 54,808 & 26.2 & 26.6 & 98.5 \\
\hline Certificate Type & & & & & \\
\hline Trade Certificate or Diploma & 1,249 & 17,621 & 22.8 & 25.7 & 89.0 \\
\hline College Certificate or Diploma & 1,691 & 37,167 & 28.7 & 27.0 & 106.1 \\
\hline Occupation & & & & & \\
\hline Managers & $286 *$ & 3,695 & $29.1 *$ & 27.6 & $105.4 *$ \\
\hline Managerial Related & $170 *$ & 3,064 & $31.4 *$ & 26.1 & $120.1 *$ \\
\hline Physical \& Life Sciences & .. & 877 & .. & 27.9 & .. \\
\hline Architecture \& Engineering & $98 *$ & 2,394 & $31.1 *$ & 28.8 & $108.1 *$ \\
\hline Math \& Computer Science &.. & 1,915 &.. & 31.1 &.. \\
\hline Social Sciences \& Religion & $161 *$ & 2,137 & $22.9 *$ & 28.5 & $80.3 *$ \\
\hline University Teaching &.. & 105 &.. & 27.7 &.. \\
\hline Other Teaching & $161 *$ & 1,732 & $25.6 *$ & 23.7 & $108.1 *$ \\
\hline Health Diagnosis & .. & 173 &.. & 31.1 & .. \\
\hline Nursing, Other Health & 420 & 9,105 & 30.0 & 30.6 & 98.0 \\
\hline Arts \& Recreation & .. & 1,539 & .. & 24.1 & .. \\
\hline Clerical & 395 & 8,967 & 21.7 & 22.3 & 97.4 \\
\hline Sales & $201 *$ & 2,614 & $20.7 *$ & 23.9 & $86.7 *$ \\
\hline Service Occupations & $299 *$ & 4,797 & $21.9 *$ & 23.8 & $91.9 *$ \\
\hline Blue Collar & 654 & 11,651 & 27.6 & 27.3 & 101.2 \\
\hline
\end{tabular}

Overall, activity-limited community college graduates earn one-and-half percent less than other graduates. Earnings differ widely by program type: the activity limited earn 11 percent less than others who took trade programs and 6 percent more in diploma and certificate programs. The gap swings widely by occupation, but the sample size in most categories is relatively small. 
Table 12. Average 1992 Earnings by Activity-Limited Status, 1990 Community College Graduates

(Full-time Workers, Earnings \$5,000 - \$500,000) - completed

\begin{tabular}{|c|c|c|c|c|c|}
\hline & \multicolumn{2}{|c|}{$\begin{array}{l}\text { Weighted } \\
\text { Number }\end{array}$} & \multicolumn{2}{|c|}{$\begin{array}{l}\text { Average } \\
\text { Earnings }\end{array}$} & \multirow[t]{2}{*}{$\begin{array}{l}\text { Earnings } \\
\text { Ratio }\end{array}$} \\
\hline & $\begin{array}{l}\text { Activity } \\
\text { Limited }\end{array}$ & $\begin{array}{c}\text { Not Activity } \\
\text { Limited }\end{array}$ & $\begin{array}{l}\text { Activity } \\
\text { Limited }\end{array}$ & $\begin{array}{c}\text { Not Activity } \\
\text { Limited }\end{array}$ & \\
\hline & & & $\$ ’ 000$ & $\$ \mathbf{S}^{\prime} 00$ & \\
\hline Industry & & & & & \\
\hline Primary Industries & .. & 1,882 & .. & 27.6 & .. \\
\hline Manufacturing \& Construction & 438 & 9,572 & 28.9 & 27.3 & 105.8 \\
\hline Transport, Communications \& Utilities & $127 *$ & 3,813 & $26.1 *$ & 29.7 & 88.2 * \\
\hline Wholesale Trade & $142 *$ & 2,140 & $25.0 *$ & 24.8 & 100.6 * \\
\hline Retail \& Consumer Services & 570 & 8,864 & 20.5 & 21.1 & 97.4 \\
\hline Finance &.. & 1,443 & .. & 23.9 & .. \\
\hline Insurance \& Real Estate & $93 *$ & 1,378 & $32.8 *$ & 25.2 & 130.3 * \\
\hline Education & $145 *$ & 1,989 & $28.7 *$ & 26.4 & 108.6 * \\
\hline Health & 725 & 13,162 & 27.4 & 29.1 & 94.3 \\
\hline Welfare \& Religion &.. & 386 &.. & 25.6 &.. \\
\hline Services to Business Management & $296 *$ & 5,061 & $23.8 *$ & 24.9 & 95.3 * \\
\hline Public Administration & $224 *$ & 5,095 & $29.0 *$ & 29.5 & 98.3 * \\
\hline Field of Study & & & & & \\
\hline Arts \& Humanities & 377 & 7,078 & 24.1 & 24.8 & 96.9 \\
\hline Health Sciences & 554 & 10,727 & 28.7 & 29.9 & 95.9 \\
\hline Other Engineering Technology & $184 *$ & 3,604 & $28.6 *$ & 28.3 & 101.3 * \\
\hline Electronics, Math \& Computer Science & $114 *$ & 3,652 & $27.9 *$ & 27.1 & 103.0 * \\
\hline Mechanical \& Structural Engineering Tech. & $192 *$ & 3,973 & $23.5 *$ & 29.4 & 80.1 * \\
\hline Natural Sciences \& Primary Industries & $207 *$ & 3,310 & $24.2 *$ & 26.5 & 91.3 * \\
\hline Social Sciences and Services & 485 & 7,249 & 26.3 & 26.3 & 100.1 \\
\hline Secretarial Sciences \& Merchandising & $156 *$ & 4,122 & $28.1 *$ & 24.0 & 117.3 * \\
\hline Management \& Administration & 508 & 8,641 & 26.2 & 23.2 & 112.6 \\
\hline Miscoded & $163 *$ & 2,453 & $22.4 *$ & 26.9 & 83.3 * \\
\hline
\end{tabular}

The earnings gap varies widely by industry and field of study, but the estimates in most instances are relatively unstable due to small sample sizes. 


\section{Earnings Models}

An individual's earnings are affected by a number of different factors, many of which are measured by the National Graduates Survey. Multivariate models are used to isolate the influence that each of a number of factors plays in a group's average earnings. A special construction of a multivariate model can be used to test the hypothesis that groups are treated differently in the labour market. ${ }^{6}$

The model divides the difference between two groups' average earnings into two components. The first is referred to as the explained or characteristics component. It captures the difference in earnings due to the differences in the earnings-related characteristics of each group. For example, if one group has more graduates with advanced degrees or who graduated from high-earnings fields of study, they are expected to earn more and this difference is captured by the characteristics component. The second component, the coefficient or residual component, picks up differences in the way in which the characteristics of each group are rewarded. If a group is treated significantly worse for a number of different characteristics, the evidence of differential treatment gets stronger.

In the interpretation of the model, a certain number of conditions must be met to provide compelling evidence of labour market discrimination. In the list that follows we describe these conditions in lay and technical terms (in brackets).

1. A model that includes the possibility of differential rewards for a designated group is better at explaining average earnings than a similar model that doesn't isolate the designated group. (An F-test for adding a designated group dummy variable and a full set of interaction terms is significant at the .05 level.)

2. The overall effect of the differential rewards for the designated group -- the coefficient component -- is negative.

3. Net of all the other characteristics, there should be a negative effect associated with being a member of the designated group identifier. (The designated group dummy variable should be negative and significant at the .05 level).

4. There should be some evidence of characteristics that are rewarded differently for which the most obvious explanation is discrimination. (There should be significant and negatively signed interaction terms that aren't easily attributable to other models of labour market behaviour.)

\footnotetext{
${ }^{6}$ The tests and rules outlined in this section are adapted from John D. Jackson and James T. Lindley, Measuring the extent of wage discrimination: a statistical test and a caveat . Applied Economics, 21, 515-540.
} 
Each of these points is addressed in the following summary tables. The inter-group earnings differential is expressed as the difference in the log of earnings for each group which indicates the percentage difference between the groups. In the first table, for example, the log earnings of visible minorities minus the log earnings of others equals .013: visible minorities earn about one-and-a-third percent more than other university graduates. The coefficient component indicates the net effect of differential treatment in the labour market. A check mark is placed beside a value that is statistically significant. So in the first table visible minorities earn just less than a percent more than others due to differing rewards, and the difference is statistically significant. The "designated group intercept" captures differences in earnings not captured by other factors. Again, its significance is denoted by a check mark. Finally, we list all variables for which the treatment was significantly different for the designated group, indicating whether the difference was positive or negative.

The model includes information on age, marital status, children, parents' education, home language, previous work experience, field of study, level of degree or length of program, public sector employment, region of residence and hours of work. The earnings differentials will be somewhat different from the previous section, since a slightly more restrictive population is used ${ }^{7}$ and missing values for each variable in the model have to be dropped. Detailed results are available upon request.

\footnotetext{
${ }^{7}$ The population is further limited to those who were employed full-time in January and October of 1991 to control for work experience since graduation.
} 


\section{V.i. Visible Minorities}

Table 13. Visible Minorities Earnings Model, 1990 University Graduates (Full-time at All Timepoints, Earnings \$5,000 - \$500,000)

\begin{tabular}{|c|c|}
\hline Differences in Log of 1992 Earnings & \\
\hline Difference between designated group and all others & 0.013 \\
\hline difference due to characteristics & 0.005 \\
\hline difference due to coefficients (1) & $0.008 \sqrt{ }$ \\
\hline Designated Group Variable & \\
\hline coefficient of designated group intercept (1) & 0.310 \\
\hline Characteristics Rewarded Differently & \\
\hline Higher Designated Group Rewards & Lower Designated Group Rewards \\
\hline Parents with Postsecondary Education & Quebec \\
\hline Home Language is French & Public Sector \\
\hline
\end{tabular}

(1) A check mark, " $\sqrt{ }$ ", indicates significance at .05 level.

Visible minority university graduates in the model earned 1.3 percent more than other graduates. The net effect of differential labour market treatment was in the favour of visible minorities. At a more detailed level, visible minorities apparently earned a premium, vis-à-vis other graduates, for Parents Postsecondary Education and Home Language - French and penalties for working in Quebec or the Public Sector (includes public administration, health, education and welfare). 
Table 14. Visible Minorities Earnings Model, 1990 Community College Graduates

(Full-time at All Timepoints, Earnings \$5,000 - \$500,000)

\begin{tabular}{|c|c|}
\hline Differences in Log of 1992 Earnings & \\
\hline Difference between designated group and all others & -0.007 \\
\hline difference due to characteristics & 0.001 \\
\hline difference due to coefficients (1) & $-0.008 \sqrt{ }$ \\
\hline Designated Group Variable & \\
\hline coefficient of designated group intercept (1) & 0.246 \\
\hline Characteristics Rewarded Differently & \\
\hline Higher Designated Group Rewards & Lower Designated Group Rewards \\
\hline & One Year Program \\
\hline & Two Year Program \\
\hline & Atlantic Provinces \\
\hline
\end{tabular}

(1) A check mark, " $\sqrt{ }$ ", indicates significance at .05 level.

Overall, visible minorities earned just under a percent less than other community college graduates, while the model estimated that they should be earning fractionally more. Although the coefficient effect is significant, the positive (but insignificant) designated group intercept indicates that any differential treatment would not be broad and systemic but confined to effects captured by the model. The model estimates that visible minorities earn smaller returns for graduating from one and two year programs and for working in the Atlantic provinces. 


\section{V.ii. Aboriginal Peoples}

Table 15. Aboriginal Peoples Earnings Model, 1990 University Graduates (Full-time at All Timepoints, Earnings \$5,000 - \$500,000)

\begin{tabular}{||lc||}
\hline Differences in Log of 1992 Earnings & 0.061 \\
Difference between designated group and all others & 0.069 \\
difference due to characteristics & -0.008 \\
difference due to coefficients (1) & \\
\hline Designated Group Variable & -0.245 \\
coefficient of designated group intercept (1) & \\
\hline Higher Designated Group Rewards & Lower Designated Group Rewards \\
\hline Full-time Experience < 1 Year & \\
\hline Characteristics Rewarded Differently & \\
\hline
\end{tabular}

(1) A check mark, " $\sqrt{ }$, indicates significance at the .05 level.

The university earnings model indicates that Aboriginal peoples earned about six percent more than others and, overall, were not treated differently in the labour market. At a more detailed level, Aboriginal peoples apparently received somewhat better-thanaverage returns for previous work experience of less than one year and somewhat lessthan-average returns to public sector employment. 
Table 16. Aboriginal Peoples Earnings Model, 1990 Community College Graduates

(Full-time at All Timepoints, Earnings \$5,000 - \$500,000)

\begin{tabular}{|c|c|}
\hline Differences in Log of 1992 Earnings & \\
\hline Difference between designated group and all others & 0.029 \\
\hline difference due to characteristics & 0.030 \\
\hline difference due to coefficients (1) & $-0.001 \sqrt{ }$ \\
\hline Designated Group Variable & \\
\hline coefficient of designated group intercept (1) & 0.402 \\
\hline Characteristics Rewarded Differently & \\
\hline Higher Designated Group Rewards & Lower Designated Group Rewards \\
\hline & One Year Program \\
\hline & Atlantic Provinces \\
\hline
\end{tabular}

(1) A check mark, " $\sqrt{ }$, indicates significance at the .05 level.

There is a very small (i.e. one-tenth of a percent) but statistically significant difference in the estimated labour market rewards for Aboriginal peoples graduates of community colleges. On the other hand, a positive but insignificant Aboriginal peoples identifier provides no indication of systematically different treatment. The model indicates that some penalty may exist for Aboriginal peoples who graduated from one-year programs or who work in the Atlantic provinces. 


\section{V.iii. Activity Limited}

Table 17. Activity-Limited Earnings Model, 1990 University Graduates

(Full-time at All Timepoints, Earnings \$5,000 - \$500,000)

\begin{tabular}{|c|c|}
\hline Differences in Log of 1992 Earnings & \\
\hline Difference between designated group and all others & -0.030 \\
\hline difference due to characteristics & 0.024 \\
\hline difference due to coefficients (1) & -0.055 \\
\hline Designated Group Variable & \\
\hline coefficient of designated group intercept (1) & 0.409 \\
\hline Characteristics Rewarded Differently & \\
\hline Higher Designated Group Rewards & Lower Designated Group Rewards \\
\hline Female & \\
\hline
\end{tabular}

(1) A check mark, " $\sqrt{ }$, indicates significance at the .05 level.

Overall, activity-limited university graduates earn three percent less than other graduates, but the model indicates the labour market does not reward them differently. 
Table 18. Activity-Limited Earnings Model, 1990 Community College Graduates

(Full-time at All Timepoints, Earnings \$5,000 - \$500,000)

\begin{tabular}{|c|c|}
\hline Differences in Log of 1992 Earnings & \\
\hline Difference between designated group and all others & -0.015 \\
\hline difference due to characteristics & 0.042 \\
\hline difference due to coefficients (1) & $-0.057 \sqrt{ }$ \\
\hline Designated Group Variable & \\
\hline coefficient of designated group intercept (1) & 0.379 \\
\hline Characteristics Rewarded Differently & \\
\hline Higher Designated Group Rewards & Lower Designated Group Rewards \\
\hline Home Language is French & Mechanical, Structural Eng. Tech. \\
\hline Children Present, June 1992 & Hours \\
\hline Two Year Program & \\
\hline Prev. Full-time Experience $<1$ Year & \\
\hline
\end{tabular}

(1) A check mark, " $\sqrt{ }$ ", indicates significance at the .05 level.

Among community college graduates, the activity limited earn a percent-and-a-half less than others, while the model estimates that they should earn about 4 percent more. The overall difference of 5.7 percent due to different treatment is significant, but the positive and insignificant activity-limited identifier provides no evidence of systemic discrimination not explicitly captured by the model. However, one of the characteristics rewarded differently is hours, indicating that activity-limited community college graduates generally earn lower wages than other graduates. The activity limited also receive lowerthan-average returns to graduating from Mechanical and Structural Engineering Technologies programs (MSE). Working in the favour of the activity limited were higher returns to French home language, children, graduating from a two-year program and previous full-time work experience less than a year. 


\section{Employment, Unemployment and Labour Force Participation}

The following tables refer to the respondent's labour force status in the week prior to the survey. The unemployment rate for a group is calculated by dividing the number of unemployed by the sum of the number of employed plus the number of unemployed. The participation rate for a group is calculated by dividing the sum of employed plus unemployed by the population of the group. Respondents for whom the labour force status or designated group status are unknown are not included in the calculations.

\section{VI.i. Visible Minorities}

Table 19. Labour Force Status (June 1992) by Visible Minority Status, 1990 University Graduates

\begin{tabular}{|c|c|c|c|c|}
\hline & \multicolumn{2}{|c|}{ "Visible Minorities } & \multicolumn{2}{|c|}{ Not Visible Minority } \\
\hline Labour Force Status & Number & Percent & Number & Percent \\
\hline Employed & 9,044 & $76.0 \%$ & 84,714 & $83.8 \%$ \\
\hline Unemployed & 1,473 & $12.4 \%$ & 9,538 & $9.4 \%$ \\
\hline Not in Labour Force & 1,387 & $11.6 \%$ & 6,780 & $6.7 \%$ \\
\hline Total & 11,903 & $100.0 \%$ & 101,032 & $100.0 \%$ \\
\hline Unemployment Rate & & $14.0 \%$ & & $10.1 \%$ \\
\hline
\end{tabular}

The employment rate for visible minorities is almost eight percentage points below the rate for other university graduates. While the majority of the difference is due to lower labour force participation by visible minorities, the unemployment rate for visible minorities is about a third higher than for other graduates. 
Table 20. Labour Force Participation Rate and Unemployment Rate (June 1992) by Visible Minority Status, 1990 University Graduates

\begin{tabular}{|c|c|c|c|c|}
\hline & \multicolumn{2}{|c|}{ Participation Rate } & \multicolumn{2}{|c|}{ Unemployment Rate } \\
\hline & $\begin{array}{l}\text { Visible } \\
\text { Minorities }\end{array}$ & $\begin{array}{c}\text { All } \\
\text { Others }\end{array}$ & $\begin{array}{c}\text { Visible } \\
\text { Minorities }\end{array}$ & $\begin{array}{c}\text { All } \\
\text { Others }\end{array}$ \\
\hline Total & $88.4 \%$ & $93.3 \%$ & $14.0 \%$ & $10.1 \%$ \\
\hline Sex & & & & \\
\hline Male & $87.1 \%$ & $93.6 \%$ & $14.4 \%$ & $10.0 \%$ \\
\hline Female & $89.7 \%$ & $93.0 \%$ & $13.6 \%$ & $10.2 \%$ \\
\hline Field of Study & & & & \\
\hline None/ Unknown & $80.8 \% *$ & $91.2 \%$ & $19.5 \% *$ & $13.8 \%$ \\
\hline Education & $96.7 \%$ & $97.3 \%$ & $11.3 \%$ & $8.0 \%$ \\
\hline Fine Arts \& Humanities & $86.0 \%$ & $89.8 \%$ & $21.2 \%$ & $12.9 \%$ \\
\hline Commerce, Economics \& Law & $94.1 \%$ & $96.8 \%$ & $11.3 \%$ & $9.8 \%$ \\
\hline Other Social Sciences & $91.6 \%$ & $91.5 \%$ & $6.6 \%$ & $12.0 \%$ \\
\hline Agricultural \& Biological Sciences & $78.6 \%$ & $85.6 \%$ & $21.8 \%$ & $12.3 \%$ \\
\hline Engineering & $86.6 \%$ & $93.4 \%$ & $15.9 \%$ & $10.3 \%$ \\
\hline Medical \& Other Health & $91.9 \%$ & $96.1 \%$ & $11.4 \%$ & $3.4 \%$ \\
\hline Math \& Physical sciences & $82.2 \%$ & $89.1 \%$ & $15.1 \%$ & $8.2 \%$ \\
\hline Province / Region & & & & \\
\hline Atlantic Provinces & $84.2 \%$ & $93.7 \%$ & $26.2 \%$ & $12.9 \%$ \\
\hline Quebec & $86.9 \%$ & $92.7 \%$ & $18.1 \%$ & $12.6 \%$ \\
\hline Ontario & $89.4 \%$ & $93.4 \%$ & $14.5 \%$ & $9.0 \%$ \\
\hline Manitoba & $83.0 \%$ & $93.0 \%$ & $10.7 \%$ & $8.2 \%$ \\
\hline Saskatchewan & $79.1 \% *$ & $93.3 \%$ & $9.3 \% *$ & $7.3 \%$ \\
\hline Alberta & $83.3 \%$ & $94.2 \%$ & $12.0 \%$ & $8.4 \%$ \\
\hline British Columbia \& Territories & $92.3 \%$ & $93.2 \%$ & $8.5 \%$ & $8.4 \%$ \\
\hline
\end{tabular}

The differences in the labour force participation and unemployment rates of visible minorities persist across almost all subgroups of university graduates. The differences are somewhat smaller for female graduates than for males. For example, the unemployment rate gap is 4.4 percentage points for men and 3.4 percentage points for women. Visible minority graduates of all fields of study except Other Social Sciences (e.g. Geography, Political Science, Sociology) have lower participation rates and higher unemployment rates. The gap between the unemployment rate of visible minorities and that of others is generally higher in eastern Canada than western Canada, virtually disappearing in British Columbia. 
Table 21. Labour Force Status (June 1992) by Visible Minority Status, 1990 Community College Graduates

\begin{tabular}{||l|rr|rr||}
\hline \hline Labour Force Status & \multicolumn{2}{|c|}{ Visible Minorities } & \multicolumn{2}{|c||}{ Not Visible Minority } \\
Employed & Number & Percent & Number & Percent \\
Unemployed & & & & \\
Not in Labour Force & 6,482 & $77.1 \%$ & 60,556 & $83.2 \%$ \\
& 1,315 & $15.6 \%$ & 8,734 & $12.0 \%$ \\
Total & 606 & $7.2 \%$ & 3,459 & $4.8 \%$ \\
& & & & \\
Unemployment Rate & 8,403 & $100.0 \%$ & 72,749 & $100.0 \%$ \\
\hline \hline
\end{tabular}

Among community college graduates, the employment rate is six percentage points lower for visible minorities than for other graduates (Table 21). More than half of the difference is due to higher unemployment rates for visible minority graduates of community colleges.

Among community college graduates, unemployment is a particularly acute problem for visible minority men (Table 22). Their unemployment rate of over 20 percent is nearly six percentage points higher than that of other male graduates. The disparity is somewhat smaller, 2.8 percentage points, among female graduates.

Visible minorities have lower unemployment rates than other community college graduates in the fields of Health Sciences, Mechanical and Structural Engineering Technologies and Natural Sciences and Primary Industries. Their unemployment rate is higher in all other fields.

The unemployment rate for visible minority community college graduates is highest in the Atlantic provinces, Quebec, Ontario and Alberta -- with particularly large gaps vis-à-vis other graduates in the latter two provinces. Visible minorities experience lower rates of unemployment than other graduates in Saskatchewan and about the same rates in Manitoba and British Columbia. 
Table 22. Labour Force Participation Rate and Unemployment Rate (June 1992) by Visible Minority Status, 1990 Community College Graduates

\begin{tabular}{|c|c|c|c|c|}
\hline & \multicolumn{2}{|c|}{ Participation Rate } & \multicolumn{2}{|c|}{ Unemployment Rate } \\
\hline & $\begin{array}{l}\text { Visible } \\
\text { Minorities }\end{array}$ & $\begin{array}{c}\text { All } \\
\text { Others }\end{array}$ & $\begin{array}{c}\text { Visible } \\
\text { Minorities }\end{array}$ & $\begin{array}{c}\text { All } \\
\text { Others }\end{array}$ \\
\hline Total & $92.8 \%$ & $95.2 \%$ & $16.9 \%$ & $12.6 \%$ \\
\hline Sex & & & & \\
\hline Male & $93.6 \%$ & $96.8 \%$ & $20.1 \%$ & $14.4 \%$ \\
\hline Female & $92.0 \%$ & $94.1 \%$ & $13.8 \%$ & $11.2 \%$ \\
\hline Field of Study & & & & \\
\hline Arts \& Humanities & $91.8 \%$ & $93.2 \%$ & $17.3 \%$ & $13.8 \%$ \\
\hline Health Sciences & $93.5 \%$ & $96.4 \%$ & $4.0 \%$ & $6.2 \%$ \\
\hline Other Engineering Technology & $94.1 \%$ & $96.2 \%$ & $24.8 \%$ & $14.7 \%$ \\
\hline Electronic, Math \& Computer Science & $90.8 \%$ & $95.8 \%$ & $21.2 \%$ & $12.9 \%$ \\
\hline Mechanical \& Structural Engineering Tech. & $96.8 \%$ & $96.3 \%$ & $15.1 \%$ & $17.0 \%$ \\
\hline Natural Sciences \& Primary Industries & $95.7 \% *$ & $96.4 \%$ & $18.1 \% *$ & $21.3 \%$ \\
\hline Social Sciences and Services & $90.6 \%$ & $95.6 \%$ & $22.2 \%$ & $11.4 \%$ \\
\hline Secretarial Sciences \& Merchandising & $91.2 \%$ & $94.6 \%$ & $14.9 \%$ & $12.0 \%$ \\
\hline Management \& Administration & $92.0 \%$ & $94.7 \%$ & $16.3 \%$ & $14.2 \%$ \\
\hline Miscoded & $96.1 \%$ & $92.8 \%$ & $29.5 \%$ & $15.7 \%$ \\
\hline Province / Region & & & & \\
\hline Atlantic Provinces & $88.6 \%$ & $96.6 \%$ & $22.8 \%$ & $19.5 \%$ \\
\hline Quebec & $92.4 \%$ & $95.2 \%$ & $15.0 \%$ & $11.6 \%$ \\
\hline Ontario & $91.4 \%$ & $94.8 \%$ & $19.4 \%$ & $12.3 \%$ \\
\hline Manitoba & $92.9 \%$ & $96.8 \%$ & $12.5 \%$ & $12.7 \%$ \\
\hline Saskatchewan & $88.2 \% *$ & $95.5 \%$ & $0.0 \% *$ & $12.6 \%$ \\
\hline Alberta & $94.2 \%$ & $94.5 \%$ & $17.2 \%$ & $9.9 \%$ \\
\hline British Columbia \& Territories & $94.9 \%$ & $95.6 \%$ & $13.5 \%$ & $13.2 \%$ \\
\hline
\end{tabular}




\section{VI.ii. Aboriginal Peoples}

Table 23. Labour Force Status (June 1992) by Aboriginal Peoples Status, 1990 University Graduates

\begin{tabular}{||l|cr|rr||}
\hline \hline & \multicolumn{2}{|c|}{ Aboriginal Peoples } & \multicolumn{2}{|c||}{ Not Aboriginal Peoples } \\
\hline Eabour Force Status & Number & Percent & Number & Percent \\
Employed & & & & \\
Unemployed & 1,320 & $84.2 \%$ & 103,062 & $82.7 \%$ \\
Not in Labour Force & $172 *$ & $10.8 \% *$ & 12,305 & $9.9 \%$ \\
Total &.. &.. & 9,310 & $7.5 \%$ \\
Unemployment Rate & 1,568 & $100.0 \%$ & 124,677 & $100.0 \%$ \\
\hline \hline
\end{tabular}

Among 1990 university graduates, both the employment and unemployment rates are slightly higher for Aboriginal peoples than for other graduates. The gap in the unemployment rate is about three percentage points for men, while female Aboriginal peoples graduates have a lower unemployment rate than other women (Table 24).

The unemployment rate for Aboriginal peoples is higher than others in about half the university fields. Small sample sizes prevent a more detailed analysis.

The Atlantic provinces and Saskatchewan have high rates of unemployment for Aboriginal peoples compared to other university graduates. In other areas of the country, the unemployment rates of Aboriginal peoples and other university graduates are more-orless equal. 
Table 24. Labour Force Participation Rate and Unemployment Rate (June 1992) by Aboriginal Peoples Status, 1990 University Graduates

\begin{tabular}{|c|c|c|c|c|}
\hline & Participa & on Rate & "Unemploy & Ient Rate \\
\hline & Aborigines & $\begin{array}{c}\text { All } \\
\text { Others }\end{array}$ & Aborigines & $\begin{array}{c}\text { All } \\
\text { Others }\end{array}$ \\
\hline Total & $95.0 \%$ & $92.5 \%$ & $11.5 \%$ & $10.7 \%$ \\
\hline Sex & & & & \\
\hline Male & $97.6 \%$ & $92.7 \%$ & $13.6 \%$ & $10.6 \%$ \\
\hline Female & $93.6 \%$ & $92.4 \%$ & $10.4 \%$ & $10.8 \%$ \\
\hline Field of Study & & & & \\
\hline Education & $97.6 \% *$ & $97.0 \%$ & $12.8 \% *$ & $8.1 \%$ \\
\hline Fine Arts \& Humanities & $100.0 \% *$ & $89.1 \%$ & $8.5 \% *$ & $14.2 \%$ \\
\hline Commerce, Economics \& Law & $100.0 \% *$ & $96.6 \%$ & $8.6 \% *$ & $9.6 \%$ \\
\hline Other Social Sciences & $94.9 \% *$ & $91.1 \%$ & $12.6 \% *$ & $12.1 \%$ \\
\hline Sciences & $83.6 \% *$ & $89.9 \%$ & $13.4 \% *$ & $9.7 \%$ \\
\hline Province / Region & & & & \\
\hline Atlantic Provinces & $100.0 \% *$ & $93.2 \%$ & $20.0 \% *$ & $13.1 \%$ \\
\hline Quebec & $90.8 \% *$ & $91.9 \%$ & $12.0 \% *$ & $12.9 \%$ \\
\hline Ontario & $100.0 \% *$ & $92.6 \%$ & $10.4 \% *$ & $10.4 \%$ \\
\hline Manitoba & $100.0 \% *$ & $92.5 \%$ & $7.3 \% *$ & $8.1 \%$ \\
\hline Saskatchewan & $92.6 \%$ & $92.8 \%$ & $18.7 \%$ & $6.4 \%$ \\
\hline Alberta & $91.6 \% *$ & $93.1 \%$ & $5.6 \% *$ & $8.7 \%$ \\
\hline British Columbia \& Territories & $92.9 \% *$ & $92.8 \%$ & $7.0 \% *$ & $8.4 \%$ \\
\hline
\end{tabular}


Table 25. Labour Force Status (June 1992) by Aboriginal Peoples Status, 1990 Community College Graduates

\begin{tabular}{||l|cr|rr||}
\hline \hline & \multicolumn{2}{|c|}{ Aboriginal Peoples } & \multicolumn{2}{c||}{ Not Aboriginal Peoples } \\
\hline Eabour Force Status & Number & Percent & Number & Percent \\
Employed & & & & \\
Unemployed & 1,796 & $70.1 \%$ & 73,381 & $83.1 \%$ \\
Not in Labour Force & 514 & $20.1 \%$ & 10,713 & $12.1 \%$ \\
Total & $251 *$ & $9.8 \% *$ & 4,239 & $4.8 \%$ \\
Unemployment Rate & 2,560 & $100.0 \%$ & 88,333 & $100.0 \%$ \\
\hline
\end{tabular}

There is a gap of 13 percentage points between the employment rate of Aboriginal peoples and other community college graduates. Most of the difference is due to the very high unemployment rate -- 22.2 percent -- of Aboriginal peoples, although their participation rate is also 5 percentage points lower than that of other graduates.

Most of the difference in the participation rates between Aboriginal peoples and other community college graduates is attributable to the low participation rates of Aboriginal women -- 87 percent of whom participated in the labour market compared to 94 percent of other female community college graduates (Table 26). Both male and female Aboriginal peoples experience much higher levels of unemployment than other community college graduates.

Unemployment rates for Aboriginal peoples are higher for all community college fields of study except Electronics, Math, Computer Science and Other Engineering Technologies, even though the participation rate is not very different in most fields.

The unemployment rate for Aboriginal peoples is at least half again as high as for other community college graduates in every region of the country. Relatively small proportions of Aboriginal peoples community college graduates participate in the labour market in Saskatchewan and Alberta -- both compared to other community college graduates in those provinces and Aboriginal peoples elsewhere in the country. 
Table 26. Labour Force Participation Rate and Unemployment Rate (June 1992) by Aboriginal Peoples Status, 1990 Community College Graduates

\begin{tabular}{|c|c|c|c|c|}
\hline & \multicolumn{2}{|c|}{ Participation Rate } & \multicolumn{2}{|c|}{ 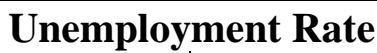 } \\
\hline & Aborigines & $\begin{array}{l}\text { All } \\
\text { Others }\end{array}$ & Aborigines & $\begin{array}{l}\text { All } \\
\text { Others }\end{array}$ \\
\hline Total & $90.2 \%$ & $95.2 \%$ & $22.2 \%$ & $12.7 \%$ \\
\hline Sex & & & & \\
\hline Male & $95.6 \%$ & $96.5 \%$ & $27.2 \%$ & $14.6 \%$ \\
\hline Female & $86.8 \%$ & $94.2 \%$ & $18.8 \%$ & $11.3 \%$ \\
\hline Field of Study & & & & \\
\hline Arts \& Humanities & $79.6 \% *$ & $93.4 \%$ & $38.0 \% *$ & $14.0 \%$ \\
\hline Health Sciences & $95.0 \%$ & $96.5 \%$ & $9.8 \%$ & $6.2 \%$ \\
\hline $\begin{array}{l}\text { Electronics, Comp Sci, Math, Other Eng. } \\
\text { Tech. }\end{array}$ & $94.4 \% *$ & $95.6 \%$ & $12.8 \% *$ & $14.2 \%$ \\
\hline Mechanical \& Structural Engineering Tech. & $95.4 \% *$ & $96.6 \%$ & $27.1 \% *$ & $17.1 \%$ \\
\hline Natural Sciences \& Primary Industries & $94.7 \% *$ & $95.8 \%$ & $33.5 \% *$ & $20.4 \%$ \\
\hline Social Sciences and Services & $87.5 \%$ & $95.3 \%$ & $19.6 \%$ & $12.1 \%$ \\
\hline Secretarial Sciences \& Merchandising & $93.9 \% *$ & $94.3 \%$ & $31.9 \% *$ & $11.3 \%$ \\
\hline Management \& Administration & $90.0 \%$ & $95.0 \%$ & $20.8 \%$ & $14.0 \%$ \\
\hline Miscoded & $83.3 \% *$ & $93.0 \%$ & $30.1 \% *$ & $17.5 \%$ \\
\hline Province / Region & & & & \\
\hline Atlantic Provinces & $94.7 \%$ & $96.5 \%$ & $31.3 \%$ & $19.3 \%$ \\
\hline Quebec & $91.2 \% *$ & $95.0 \%$ & $23.7 \% *$ & $11.5 \%$ \\
\hline Ontario & $91.9 \%$ & $94.6 \%$ & $18.9 \%$ & $12.8 \%$ \\
\hline Manitoba & $94.0 \%$ & $96.4 \%$ & $32.3 \%$ & $11.2 \%$ \\
\hline Saskatchewan & $85.1 \%$ & $96.5 \%$ & $36.5 \%$ & $10.5 \%$ \\
\hline Alberta & $75.1 \%$ & $95.3 \%$ & $16.8 \%$ & $10.8 \%$ \\
\hline British Columbia \& Yukon & $96.0 \%$ & $95.8 \%$ & $20.0 \%$ & $13.5 \%$ \\
\hline Northwest Territories & $92.3 \%$ & $98.0 \%$ & $20.9 \%$ & $3.3 \%$ \\
\hline
\end{tabular}


VI.iii. Activity Limited

Table 27. Labour Force Status (June 1992) by Activity-Limited Status, 1990 University Graduates

\begin{tabular}{||l|rr|rr||}
\hline \hline & \multicolumn{2}{|c|}{ Activity Limited } & \multicolumn{2}{c||}{ Not Activity Limited } \\
\hline Labour Force Status & Number & Percent & Number & Percent \\
Employed & 3,757 & $76.8 \%$ & 100,625 & $82.9 \%$ \\
Unemployed & 614 & $12.6 \%$ & 11,863 & $9.8 \%$ \\
Not in the Labour Force & 522 & $10.7 \%$ & 8,868 & $7.3 \%$ \\
Unemployment Rate & & $14.1 \%$ & & $10.5 \%$ \\
& & & & \\
\hline \hline
\end{tabular}

The employment rate for university graduates with activity limitations is six percentage points lower than for other graduates. While a smaller proportion of the activity limited participate in the labour force, their unemployment rate is about one-third higher than that of other graduates. The differences are greater for women than men: the participation and unemployment rates gap are about five percentage points for women and one-and-a-half percentage points for men (Table 28).

The unemployment rates of activity-limited university graduates vary greatly by field of study. While the unemployment rate for the activity limited is higher than for other university graduates in most fields, their rate is lower for graduates of Engineering, Medical and Other Health and Commerce, Economics and Law.

The activity limited experience higher unemployment rates than other university graduates in every province except Manitoba and Alberta. 
Table 28. Labour Force Participation Rate and Unemployment Rate (June 1992) by Activity-Limited Status, 1990 University Graduates

\begin{tabular}{|c|c|c|c|c|}
\hline & Participa & on Rate & Unemploy & nent Rate \\
\hline & $\begin{array}{l}\text { Activity } \\
\text { Limited }\end{array}$ & $\begin{array}{c}\text { All } \\
\text { Others }\end{array}$ & $\begin{array}{l}\text { Activity } \\
\text { Limited }\end{array}$ & $\begin{array}{c}\text { All } \\
\text { Others }\end{array}$ \\
\hline Total & $89.3 \%$ & $92.7 \%$ & $14.1 \%$ & $10.5 \%$ \\
\hline Sex & & & & \\
\hline Male & $91.4 \%$ & $92.8 \%$ & $12.3 \%$ & $10.5 \%$ \\
\hline Female & $87.7 \%$ & $92.6 \%$ & $15.5 \%$ & $10.6 \%$ \\
\hline Field of Study & & & & \\
\hline None/ Unknown & $95.0 \% *$ & $90.2 \%$ & $27.7 \% *$ & $13.9 \%$ \\
\hline Education & $93.9 \%$ & $97.1 \%$ & $8.8 \%$ & $8.2 \%$ \\
\hline Fine Arts \& Humanities & $89.9 \%$ & $89.2 \%$ & $19.2 \%$ & $13.8 \%$ \\
\hline Commerce, Economics \& Law & $94.0 \%$ & $96.7 \%$ & $6.2 \%$ & $9.7 \%$ \\
\hline Other Social Sciences & $82.0 \%$ & $91.7 \%$ & $18.5 \%$ & $11.8 \%$ \\
\hline Agricultural \& Biological Sciences & $75.1 \% *$ & $84.0 \%$ & $48.2 \% *$ & $13.8 \%$ \\
\hline Engineering & $92.9 \% *$ & $92.0 \%$ & $6.0 \% *$ & $11.2 \%$ \\
\hline Medical \& Other Health & $93.4 \% *$ & $95.2 \%$ & $0.9 \% *$ & $4.9 \%$ \\
\hline Math \& Physical sciences & $87.0 \% *$ & $87.4 \%$ & $12.7 \% *$ & $9.4 \%$ \\
\hline Province & & & & \\
\hline Newfoundland & $91.9 \%$ & $95.2 \%$ & $18.2 \%$ & $12.8 \%$ \\
\hline Prince Edward Island & $100.0 \% *$ & $98.8 \%$ & $30.0 \% *$ & $16.4 \%$ \\
\hline Nova Scotia & $87.3 \%$ & $91.6 \%$ & $16.6 \%$ & $11.4 \%$ \\
\hline New Brunswick & $96.5 \% *$ & $94.1 \%$ & $21.1 \% *$ & $14.7 \%$ \\
\hline Quebec & $86.4 \%$ & $92.1 \%$ & $17.9 \%$ & $12.8 \%$ \\
\hline Ontario & $89.6 \%$ & $92.7 \%$ & $16.2 \%$ & $10.1 \%$ \\
\hline Manitoba & $89.4 \%$ & $92.9 \%$ & $7.2 \%$ & $8.2 \%$ \\
\hline Saskatchewan & $84.8 \% *$ & $93.2 \%$ & $11.1 \% *$ & $6.9 \%$ \\
\hline Alberta & $91.8 \%$ & $93.2 \%$ & $6.0 \%$ & $8.9 \%$ \\
\hline British Columbia \& Territories & $89.2 \%$ & $93.0 \%$ & $11.4 \%$ & $8.3 \%$ \\
\hline
\end{tabular}


Table 29. Labour Force Status (June 1992) by Activity-Limited Status, 1990 Community College Graduates

\begin{tabular}{||l|rr|rr||}
\hline \hline & \multicolumn{3}{|c|}{ Activity Limited } & \multicolumn{2}{|c||}{ Not Activity Limited } \\
\hline Labour Force Status & Number & Percent & Number & Percent \\
Employed & 4,187 & $71.1 \%$ & 70,989 & $83.5 \%$ \\
Unemployed & 955 & $16.2 \%$ & 10,272 & $12.1 \%$ \\
Not in the Labour Force & 744 & $12.6 \%$ & 3,745 & $4.4 \%$ \\
& & & & \\
Unemployment Rate & & $18.6 \%$ & & $12.6 \%$ \\
\hline \hline
\end{tabular}

There is a gap of more than 12 percentage points between the employment rates of activity-limited and other community college graduates. While two-thirds of this gap is due to lower labour force participation among the activity limited, that group also has a 50 percent higher unemployment rate.

In contrast to most of the other groups examined, the participation rate of activitylimited men is lower than the female rate among community college graduates. The rates for both genders are well below the corresponding rates for other community college graduates. Similarly, men with activity limitations have a higher unemployment rate and a larger gap vis-à-vis other graduates than is the case for female community college graduates with activity limitations.

Activity-limited community college graduates have higher unemployment rates and lower participation rates than other graduates within every field of study.

The participation rate for activity-limited community college graduates is lower than for other graduates in every province, with particularly large differences in Ontario, New Brunswick and Quebec. The activity limited experienced higher rates of unemployment than other community college graduates in all provinces but New Brunswick, Alberta and Northwest Territories. 
Table 30. Labour Force Participation Rate and Unemployment Rate (June 1992) by Activity-Limited Status, 1990 Community College Graduates

\begin{tabular}{|c|c|c|c|c|}
\hline & Participat & on Rate & Unemploy & ient Rate \\
\hline & $\begin{array}{l}\text { Activity } \\
\text { Limited }\end{array}$ & $\begin{array}{c}\text { All } \\
\text { Others }\end{array}$ & $\begin{array}{l}\text { Activity } \\
\text { Limited }\end{array}$ & $\begin{array}{c}\text { All } \\
\text { Others }\end{array}$ \\
\hline Total & $87.4 \%$ & $95.6 \%$ & $18.6 \%$ & $12.6 \%$ \\
\hline Sex & & & & \\
\hline Male & $86.8 \%$ & $97.2 \%$ & $22.9 \%$ & $14.4 \%$ \\
\hline Female & $87.9 \%$ & $94.4 \%$ & $14.8 \%$ & $11.3 \%$ \\
\hline Field of Study & & & & \\
\hline Arts \& Humanities & $81.0 \%$ & $94.1 \%$ & $17.7 \%$ & $14.2 \%$ \\
\hline Health Sciences & $92.3 \%$ & $96.7 \%$ & $10.7 \%$ & $6.0 \%$ \\
\hline Other Engineering Technology & $89.5 \% *$ & $96.0 \%$ & $26.2 \% *$ & $14.3 \%$ \\
\hline Electronic, Math \& Computer Science & $78.4 \% *$ & $96.4 \%$ & $16.6 \% *$ & $13.3 \%$ \\
\hline Mechanical \& Structural Engineering Tech. & $86.2 \%$ & $97.3 \%$ & $27.2 \%$ & $16.8 \%$ \\
\hline Natural Sciences \& Primary Industries & $85.1 \%$ & $96.6 \%$ & $22.5 \%$ & $20.8 \%$ \\
\hline Social Sciences and Services & $89.7 \%$ & $95.5 \%$ & $15.4 \%$ & $12.1 \%$ \\
\hline Secretarial Sciences \& Merchandising & $88.9 \% *$ & $94.6 \%$ & $15.6 \% *$ & $11.5 \%$ \\
\hline Management \& Administration & $86.9 \%$ & $95.3 \%$ & $18.5 \%$ & $13.9 \%$ \\
\hline Miscoded & $92.9 \%$ & $92.7 \%$ & $33.1 \%$ & $16.4 \%$ \\
\hline Province / Region & & & & \\
\hline Newfoundland & $93.4 \%$ & $96.5 \%$ & $27.8 \%$ & $21.7 \%$ \\
\hline Prince Edward Island & $87.9 \% *$ & $97.2 \%$ & $35.6 \% *$ & $14.7 \%$ \\
\hline Nova Scotia & $91.8 \%$ & $94.9 \%$ & $21.3 \%$ & $17.7 \%$ \\
\hline New Brunswick & $84.0 \%$ & $97.9 \%$ & $15.8 \%$ & $19.6 \%$ \\
\hline Quebec & $85.9 \%$ & $95.2 \%$ & $13.6 \%$ & $11.6 \%$ \\
\hline Ontario & $80.7 \%$ & $95.5 \%$ & $20.6 \%$ & $12.4 \%$ \\
\hline Manitoba & $90.7 \%$ & $96.7 \%$ & $19.8 \%$ & $12.0 \%$ \\
\hline Saskatchewan & $90.8 \%$ & $96.0 \%$ & $22.2 \%$ & $11.5 \%$ \\
\hline Alberta & $93.1 \%$ & $94.7 \%$ & $9.8 \%$ & $11.1 \%$ \\
\hline British Columbia \& Yukon & $93.1 \%$ & $96.0 \%$ & $20.0 \%$ & $13.1 \%$ \\
\hline Northwest Territories & $89.3 \% *$ & $95.3 \%$ & $0.0 \% *$ & $12.6 \%$ \\
\hline
\end{tabular}




\section{Why are fewer designated group members employed than other graduates?}

Most categories of designated group graduates have lower participation rates and higher unemployment rates than other graduates. Before discussing the possibility of discrimination to explain these results, we explore some alternative explanations.

\section{Visible Minorities}

More visible minorities may have been visa students and therefore ineligible to seek work in Canada.

A larger proportion of visible minorities were indeed visa students. However, the employment rates for former visa students do not differ greatly from other students -- the employment and unemployment gap for visible minorities exists within both groups.

Since more visible minority graduates are former visa students and a higher proportion of visible minorities are recent immigrants, their language skills in English or French may lag those of other graduates.

To start with, the fact that those with foreign mother tongues or home languages have graduated from Canadian postsecondary institutions limits the validity of this proposition. Empirically, a slightly higher proportion of visible minorities do have mother tongues or home languages other than English or French. But again an employment gap exits between visible minorities and others in every category. In fact, the group with the highest employment rate is comprised of those with a mother tongue other than English or French who are not in a visible minority group.

\section{Aboriginal Peoples}

A greater percentage of Aboriginal Peoples may live in areas where jobs are scarce(e.g. reserves or other non-urban areas), thereby increasing their unemployment rate vis-à-vis groups who are concentrated in larger labour markets.

There may be some merit to this proposition, but it can't be readily tested with the National Graduates Surveys which only contain data at the provincial level. Remember that Aboriginal peoples graduates of universities, which tend to be in larger centres, have similar employment rates to other graduates.

\section{Activity Limited}

Those with severe activity limitations may be less employable than those with relatively minor limitations and could therefore account for much of the unemployment gap vis-àvis other graduates.

One might argue, on the other hand, that if someone is able to complete postsecondary studies and look for work then they should be sufficiently able to perform a range of paid jobs. Never-the-less, we constructed an index based on 
the number of activity limitations cited by respondents -- work, home, school and other -- and the presence of a long-term disability or handicap. While the participation rate tended to fall with the index of limitation, there was no clear correlation between the index value and the unemployment rate. Respondents who reported any level of activity limitation, according to this index, had higher unemployment rates than those with no limitations. Thus severity of limitation appears to have an impact on the participation rate, but merely the presence of any limitation triggers an increase in the unemployment rate.

In summary, no single factor that can be tested with the National Graduates Survey readily accounts for the elevated unemployment rates of graduates in most designated groups. To examine the possibility that hiring discrimination played a role in the elevated unemployment rates for these groups, we tested a model of the probability of being employed similar to the earnings model described in the previous section. Although the non-linear model used to estimate the probability of being employed is more restrictive than the linear model used to estimate earnings, it does allow us to test some of the same propositions. ${ }^{8}$

Similar to the earnings analyses, a model that doesn't allow for the differential treatment is compared to one that does. A statistical test is used to determine whether, overall, the designated group is rewarded differently in terms of the probability of employment. The target group identifier variable captures effects not explicitly included in the model and is therefore an indicator of systemic bias. Other variables in the model point to characteristics that are rewarded differently for members of the target group.

We tested the employment model on visible minorities versus others among university graduates, since they provided the largest sample size. Sample size was important, since we felt it appropriate to run separate models for men and women. ${ }^{9}$ The models estimated more substantial negative employment impacts for visible minority men than visible minority women.

For both men and women, the model estimated statistically significant overall employment rewards for visible minority versus other graduates. However for women, the effect was much smaller. Furthermore there was no single characteristic for which

\footnotetext{
${ }^{8}$ A logistic regression model was used to estimate the probability of employment versus unemployment. Due to the shape of the logistic function, the average probability does not decompose into the sum of the characteristics and residual components. Logistic regression employs a maximum likelihood estimator (MLE) that requires convergence criteria be met through successive iterations. We tested models for Aboriginal peoples and the activity limited, as well as visible minorities, but abandoned them when convergence could not be achieved within 500 iterations.

${ }^{9}$ There are two reasons to run models for each gender. First, the unemployment gap is smaller for women than men. Second, another study by the same authors (The Gender Earnings Gap Among Recent Postsecondary Graduates, 1984-92) indicates that women interact differently with the labour market than men.
} 
visible minority women were rewarded significantly worse than other female graduates. The indicator variable for visible minority in the female model was negative but not statistically significant. Therefore the model provides scant evidence of hiring discrimination for visible minority women compared to other female university graduates.

In the male employment model, there were a number of significant negative factors. Visible minorities received lower employment returns to being married or divorced, to having a mother tongue other than English or French and, particularly, to graduating from science-based fields of study (Agriculture \& Biology, Engineering, Medical \& Other Health and Math \& Physical Sciences). On the other hand, visible minorities received significantly higher employment returns to graduating with a $\mathrm{PhD}$ and having previous full-time work experience of greater than a year. The identifier variable was positive but not statistically significant. Thus evidence of differential hiring treatment is strongest for visible minority graduates of university science programs, with weaker language and marital status effects.

Given the results of the employment model, hiring discrimination cannot be ruled out as an explanation for the higher unemployment rates of visible minority university graduates -- particularly graduates of science programs. But it is hard to envision how a classical model of discrimination would produce the results presented in the paper. Employment and wage discrimination must be supported by an employer and customer preference for dealing with members of one group. Customer complicity is required, since non-discriminating employers should be able to hire members of the disadvantaged group at lower wages. Unless customers clearly prefer to deal only with discriminating firms, competitive forces would then work to the advantages of nondiscriminating firms -eventually putting upward pressure on the employment rate and wages of the disadvantaged group. Why then would employment discrimination be focused on the graduates of science-based programs? And why would visible minority graduates be earning the same or more than other graduates when their unemployment rate is much higher?

An alternative interpretation is related to tacit hiring quotas ${ }^{10}$. If employers hire visible minority graduates in relation to their overall labour market representation, science graduates will be underrepresented. Remember that visible minorities receive a high proportion of science degrees in relation to their representation among university graduates and the population as a whole. Thus adherence to broadly defined quotas could have detrimental effects for visible minority graduates of science programs. That is not to say that blind adherence to hiring quotas does not constitute a form of discrimination -particularly if quotas are treated as ceilings. However, if employers had discriminatory tendencies, one would expect to see it in differential earnings growth over time. This does not appear to be the case for visible minority university graduates.

\footnotetext{
${ }^{10}$ The authors are not aware of any explicit hiring quotas under Canadian legislation, but employers may well adopt tacit quotas if their employment equity performance is subject to legislative or quasi-judicial review.
} 
We ran the same earnings model presented earlier, using data from the 1991 Follow-up of Graduates Survey. This survey was a re-interview of 1986 graduates five years after graduation. Overall, earnings for visible minorities were essentially the same as for other university graduates. Earnings for visible minorities were higher in all four of the science fields, significantly so in two of the fields -- Engineering and Medical \& Other Health. In fact, this is what one would expect if visible minorities and others were being treated equally after being hired, since visible minority graduates who did make it in probably had better-than-average qualifications and perceived capabilities to begin with. Thus, on average, employers do not seem to discriminate against visible minority graduates already in their employ.

On the other hand, if employers were perfectly rational and non-discriminatory, one might expect that the high relative performance of visible minority graduates (assuming earnings are a measure of performance) would eventually result in increased hiring proportions for this group. This also, does not seem to be the case. 


\section{Summary and Discussion}

Throughout this report the results have been organized primarily by the type of analysis with the numbers for each designated group presented in succession. While some of the findings were similar for each group, there were some significant differences.

Therefore, we thought it appropriate to conclude by summarizing and discussing all of the results for each group separately.

\section{Visible Minorities}

Visible minorities fared well in terms of their representation in the 1992 graduating class of Canadian universities and community colleges. Visible minorities comprised just over 10 percent of the graduates at each type of institution compared to their nine-and-a-half percent share of the 1991 population. At the universities, the representation of visible minorities increased with degree level -- from 10 percent at the undergraduate level to just over 19 percent at the $\mathrm{PhD}$ level. Members of visible minorities tended to be concentrated in science-based fields of studies at the universities, but the same pattern was not evident at community colleges.

The earnings of visible minority graduates did not differ greatly from the earnings of other graduates. Among full-time workers, visible minority university graduates earned an average of two percent more than other graduates; visible minority community college graduates earned two percent less. The relative earnings of visible minority university graduates fell with each step up in degree level -- from 102.5 at the undergraduate level to 98.7 at the masters level to 86.5 at the $\mathrm{PhD}$ level.

Even though the earnings of visible minorities were nearly the same as the earnings of other graduates, the possibility remains that they are rewarded differently in the labour market. Accordingly, we used a multivariate earnings model to estimate whether visible minorities were rewarded the same as other graduates. While the model results were statistically significant, the net effect accounted for less than one percent of average earnings. The effect was positive (i.e. better than others) for university graduates and negative for community college graduates. In terms of earnings, then, both the descriptive and multivariate analyses indicated negligible differences between visible minorities and other graduates.

In contrast to the earnings results, the employment patterns of visible minorities differ substantially from those of other graduates. Visible minorities have low participation rates and high unemployment rates relative to other graduates. These differences are evident for graduates of most fields of study and occur in most regions, the main exception being British Columbia. Since these results raise the possibility of hiring discrimination against visible minority members, we tested a multivariate model of employment (versus unemployment) for the university graduates. The model indicated 
that for women, the employment differences between visible minorities and others were not that great. On the other hand, the model showed negative employment effects for visible minority men, particularly if they had graduated from science-based fields of study.

Several explanations for the high rate of unemployment for visible minorities were explored, none of which seemed fully satisfactory. Since the elevated unemployment rates were widespread, hiring discrimination based on employers' and customers' preferences may be a factor. But such a form of discrimination would probably lead to lower earnings for the disadvantaged group and that is not the case for visible minorities. An alternative explanation relates to tacit hiring quotas: if employers blindly hire visible minorities in relation to their population share, visible minority university graduates -- particularly in science-based fields -- will be underrepresented. While this explanation fits better with the earnings patterns, it too is not fully satisfactory. Longer term data indicate that earnings grow faster for visible minority graduates -- what one might expect given the statistically more selective hiring pattern. Why, then, wouldn't employers adjust their hiring patterns to reflect the better performance (assuming wage growth is a measure of performance) of visible minority employees? In all probability, some elements of discrimination and quota distortion contribute to the elevated unemployment rate of visible minorities. Unfortunately, the relative strength of these effects cannot be directly estimated with the NGS data.

\section{Aboriginal Peoples}

The representation rate of Aboriginal peoples is much lower among 1990 university graduates, at 1.2 percent, than among community college graduates, 2.8 percent. Both figures are lower than the Aboriginal peoples share of the 1991 population, 3.8 percent, and their share of the workforce, 3.0 percent. Among university graduates, the representation rate is particularly low for advanced degree holders. Provincially, Aboriginal peoples were best represented among the graduates of Saskatchewan and Manitoba universities. Two-thirds of the community college graduates in the Northwest Territories identified themselves as Aboriginal peoples.

Aboriginal peoples earned, on average, about the same as other graduates. The multivariate model estimated that Aboriginal peoples were not treated differently than other university graduates in terms of earnings. While there was a statistically significant effect for community college graduates, it amounted to only a tenth of a percent of the average earnings. Thus it would be hard to conclude that there is any discernible level of earnings discrimination for Aboriginal peoples in the class of 1990.

The employment situation is very different for Aboriginal peoples who graduated from universities than for those who attended community colleges. Among university graduates, Aboriginal peoples had a slightly lower participation rate and a slightly higher unemployment rate than others in their class. The unemployment rate for Aboriginal peoples was relatively high for men and those living in Saskatchewan and the Atlantic 
provinces. Elsewhere in the country and among women, Aboriginal peoples had unemployment rates equal to or less than other graduates.

In contrast to the university graduates, there is a pervasive gap between the unemployment situation for Aboriginal peoples and other community college graduates. Overall, the unemployment rate for Aboriginal peoples is almost ten percentage points higher than for other graduates. This gap persists in all regions and within all fields of study expect one (the combination of Electronics, Math, Computer Science and Other Engineering Technologies).

Why should the unemployment rate of Aboriginal peoples be so high compared to other community college graduates or Aboriginal peoples who graduated from universities? We can think of several possible explanations, none of which can be fully addressed with the NGS data. First, Aboriginal peoples graduates of community colleges may be more concentrated in non-urban areas, particularly reserves, with higher local unemployment rates than are other community college graduates. Unfortunately, the NGS does not contain the geographical detail to test this hypothesis. Aboriginal peoples graduating from universities may not be affected for several reasons. First, universities are usually located in larger centres with a greater range of job opportunities. Secondly, the number of Aboriginal peoples graduating from universities is so small in relation to the population that the demand for public sector professionals and administrators, even in rural locations, provides relatively more opportunities for this group. The NGS does have some evidence to support this: 63 percent of Aboriginal-peoples university graduates are employed in the public sector compared to 52 percent of other university graduates and 55 percent of Aboriginal peoples community college graduates.

The data do not allow us to overlook the possibility of hiring discrimination against Aboriginal peoples. However, the fact that Aboriginal peoples earn, on average, the same as other graduates limits this argument to some extent. It is also hard to argue that implicit quotas would dampen the employment opportunities for highly-qualified Aboriginal peoples, since their representation among postsecondary graduates is lower than their proportion of the population. Unfortunately, we were unable to fit a model of employment to shed any further light on this issue.

\section{Activity Limited}

We employ the term "activity limited" as opposed to "persons with disabilities" due to the differences in the screening sequences used in the NGS and the Employment Equity Data Program (see Appendix A.). The activity limited comprise just under four percent of 1990 university graduates and six-and-a-half percent of community college graduates. The Employment Equity Data Program estimates that persons with disabilities comprised seven percent of the total population in 1991 and six-and-a-half percent of the workforce. 
Thus, if "activity limited" closely approximates "persons with disabilities", this group is underrepresented at universities and proportionately represented at community colleges. The only point that stands out in relation to the distribution of activity-limited graduates is the low representation rate among graduates of Quebec universities and community colleges.

Activity-limited university graduates earn about seven percent less than others in their class. The gap for community college graduates is only one-and-a-half percent. The earnings models did not generate any strong evidence of earnings discrimination towards activity-limited graduates.

Activity-limited graduates have lower labour force participation rates and higher unemployment rates than other graduates, with the differences being somewhat larger in the community college class. While the participation rate of activity-limited graduates was lower in all regions of the country and across all fields of study, the unemployment rate was not consistently higher. Activity-limited university graduates in several fields of study had lower unemployment rates than other graduates. In Alberta, the unemployment rates of activity-limited graduates of universities and community colleges were both lower than the rates of other graduates. In Manitoba, the unemployment rate of activity-limited university graduates was lower.

We explored the notion that the low participation rate and high unemployment rate of activity-limited graduates may be related to the severity of the limitation. While the participation rate falls off as a simple index of severity rises, the unemployment rate remains fairly constant. Although the results do not rule out hiring discrimination, some may argue that it is not so much discrimination as a perception of added employment costs that dampens the job opportunities for the activity limited. One might also suppose that the job search patterns may differ for the activity limited. In either case, public policy remedies may be appropriate since measurable costs are associated with providing increased access for persons with disabilities and providing any necessary assistance to increase the efficiency of their job search patterns. One might even examine whether there is a set of policies in Alberta that accounts for the relatively low unemployment rate of activity-limited graduates in that province. 


\section{Appendix A.}

\section{Identification Questions}

\begin{tabular}{|c|c|}
\hline 1992 NGS & Employment Equity Data Program \\
\hline $\begin{array}{l}\text { Visible Minorities } \\
\text { Canadians come from many ethnic, } \\
\text { cultural or racial backgrounds; for } \\
\text { example, British, French, North American } \\
\text { Indian, Chinese, Black, Japanese or } \\
\text { Greek. What is your ethnic or cultural } \\
\text { background? } \\
\text { Any single response or element of a } \\
\text { multiple response corresponding to the } \\
\text { following list was coded as a visible } \\
\text { minority. } \\
\text { - Blacks } \\
\text { - South Asians } \\
\text { - Chinese } \\
\text { - Koreans } \\
\text { - Japanese } \\
\text { - South East Asians } \\
\text { - Filipinos } \\
\text { - Other Pacific Islanders } \\
\text { - West Asians and Arabs } \\
\text { - Latin Americans }\end{array}$ & $\begin{array}{l}\text { Any single response or element of a } \\
\text { multiple response corresponding to the } \\
\text { following list was coded as a visible } \\
\text { minority. } \\
\text { - Blacks } \\
\text { - South Asians } \\
\text { - Chinese } \\
\text { - Koreans } \\
\text { - Japanese } \\
- \text { South East Asians } \\
\text { - Filipinos } \\
\text { - Other Pacific Islanders } \\
\text { - West Asians and Arabs } \\
\text { - Latin Americans } \\
+ \text { Ethnicity and Birthplace } \\
+ \text { Mother tongue } \\
+ \text { Ethnicity write-ins }\end{array}$ \\
\hline $\begin{array}{l}\text { Aboriginal Peoples } \\
\text { Identified through the ethnicity question } \\
-\quad \text { North American Indian } \\
-\quad \text { Inuit } \\
-\quad \text { Métis }\end{array}$ & $\begin{array}{l}\text { - } \begin{array}{l}\text { Aboriginal Peoples } \\
\text { Identified through the ethnicity question }\end{array} \\
-\quad \text { North American Indian } \\
-\quad \text { Inuit } \\
-\quad \text { Métis } \\
-\quad \text { Specific First Nation or Band Name }\end{array}$ \\
\hline
\end{tabular}




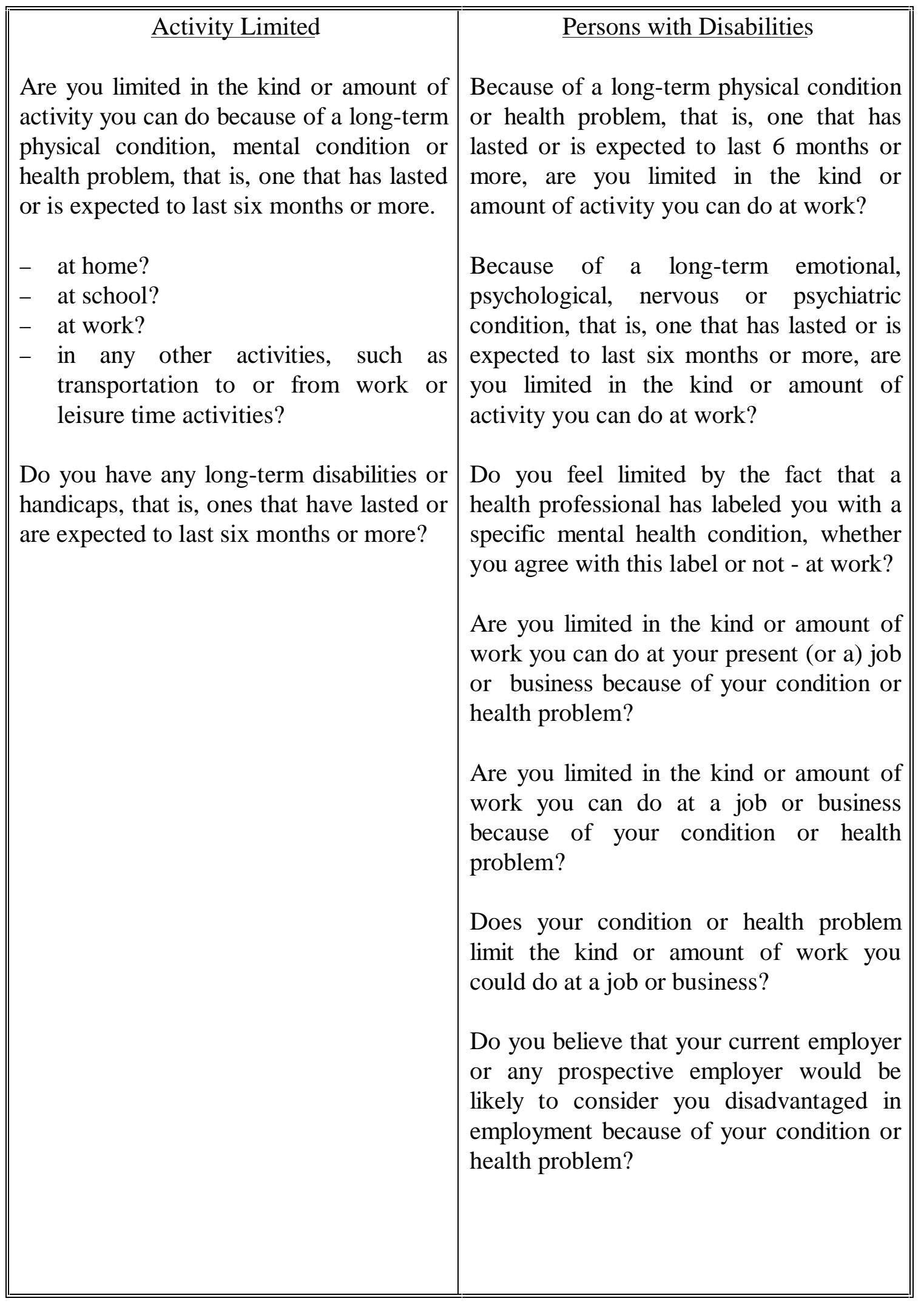




\begin{tabular}{|c|c|}
\hline Activity Limited (continued) & $\begin{array}{l}\text { Persons with Disabilities (continued) } \\
\text { Because of your condition or health } \\
\text { problem would you require any of the } \\
\text { following to be able to work? } \\
\text { i) Human support such as: } \\
\text { reader, oral or sign language interpreter, } \\
\text { job coach personal assistant } \\
\text { ii) Technical aids and devices such as: } \\
\text { voice synthesizer, telecommunications } \\
\text { device for the deaf (TDD), infrared } \\
\text { system, computer } \\
\text { iii) Communication services such as: } \\
\text { conversion of print to Braille, to audio } \\
\text { tape, to enlarged print } \\
\text { iv) Job redesign (modified or different } \\
\text { duties) } \\
\text { v) Modified hours or days or reduced } \\
\text { work hours } \\
\text { vi) Accessible transportation } \\
\text { vii) Other, please specify } \\
\text { Do you require modified features or } \\
\text { arrangements at your workplace, such as } \\
\text {.. } \\
\text { i) Handrails, ramps } \\
\text { ii) Appropriate parking } \\
\text { iii) Accessible elevator } \\
\text { iv) Accessible workstation } \\
\text { v) Accessible washrooms } \\
\text { vi) Other, please specify } \\
\text { In the past five years, do you believe that } \\
\text { because of your condition or health } \\
\text { problem, you have been refused: } \\
\text { (a) employment } \\
\text { (b) a promotion } \\
\text { (c) access to training programs, or } \\
\text { (d) has your employment been terminated } \\
\text { Does your condition or health problem } \\
\text { completely prevent you from working at a } \\
\text { job or business? }\end{array}$ \\
\hline
\end{tabular}




\begin{tabular}{|c|c|}
\hline Activity Limited (continued) & $\begin{array}{l}\text { Persons with Disabilities (continued) } \\
\text { Some people have encountered barriers } \\
\text { which have discouraged them from } \\
\text { looking for work. Could you think about } \\
\text { your own situation and indicate which of } \\
\text { the following situations might apply to } \\
\text { you? Please answer yes or no to each of } \\
\text { the statements. } \\
\text { i) You would lose some or all of your } \\
\text { current income if you went to work. } \\
\text { ii) You would lose some or all of your } \\
\text { current additional support such as your } \\
\text { drug plan or housing if you went to work. } \\
\text { iii) Your family or friends have } \\
\text { discouraged your going to work. } \\
\text { iv) Family responsibilities prevent you. } \\
\text { v) Information about jobs is not } \\
\text { accessible to you. } \\
\text { vi) You worry about being isolated by } \\
\text { other workers on the job. } \\
\text { vii) You have been the victim of } \\
\text { discrimination. } \\
\text { adequate. } \\
\text { ix) Lack of accessible transportation. } \\
\text { ingailable. }\end{array}$ \\
\hline
\end{tabular}




\section{Appendix B.}

\section{Industry and Occupation Coding}

Industry Description

Managers

Managerial Related

Physical \& Life Sciences

Architecture \& Engineering

Math \& Computer Science

Social Sciences \& Religion

University Teaching

Other Teaching

Health Diagnosis

Nursing, Other Health

Arts \& Recreation

Clerical

Sales

Service Occupations

Blue Collar

Not Specified

Occupation Description

Primary Industries

Manufacturing \& Construction

Transport, Communications \& Utilities

Wholesale Trade

Retail \& Consumer Services

Finance

Insurance \& Real Estate

Education

Health

Welfare \& Religion

Services to Business Management

Public Administration

Not Specified
Standard Occupation Classification (1980)

Codes

$1111-1158$

$1171-1179$

$2111-2139$

$2141-2169$

$2181-2189$

$2311-2519$

$2711-2719$

$2731-2799$

$3111-3119$

$3130-3169$

$3311-3379$

$4110-4199$

$5130-5199$

$6111-6199$

7113 - 9599

Less than 1111 or 9910 - 9919

Standard Industrial Classification (1980)

Codes

$001-092$

$101-449$

$451-499$

$501-599$

601 - $692 \&$ 911- 999

$701-729 \& 741-749$

$731-733 \& 751-761$

$851-859$

$861-866$

$867-869 \& 981$

$771-779$

$811-841$

Greater than 999 